\title{
Business Cycles and Compositional Variation in U.S. Unemployment
}

\author{
Jaap H. Abbring * \\ Gerard J. van den Berg ${ }^{\dagger}$ \\ Jan C. van Ours ${ }^{\ddagger}$
}

April 24, 1997

\begin{abstract}
In the past decades several features of U.S. unemployment dynamics have been investigated empirically. The original focus of research was on the duration of unemployment. In later studies the cyclicality of incidence and duration, compositional effects and duration dependence of the exit rate out of unemployment have been investigated. Unlike the partial approach of previous studies this paper takes all elements of unemployment dynamics simultaneously into account. We find that cyclical fluctuations in unemployment are driven by variations in the incidence, individual exit probabilities and the composition of the inflow into unemployment. We also find negative duration dependence of the unemployment exit rate which can be attributed to employers ranking workers according to the length of their unemployment spell.
\end{abstract}

\footnotetext{
*Department of Economics, University of Amsterdam, Roetersstraat 11, NL-1018 WB Amsterdam, The Netherlands, and Tinbergen Institute.

${ }^{\dagger}$ Department of Economics, Free University Amsterdam, De Boelelaan 1105, NL-1081 HV Amsterdam, The Netherlands, Tinbergen Institute and CEPR.

${ }_{\ddagger}^{\ddagger}$ Erasmus University Rotterdam, Tinbergen Institute, Burgemeester Oudlaan 50, NL-3062 PA Rotterdam, The Netherlands and CEPR.
}

Keywords: unemployment composition, unemployment duration, business cycle, sorting, ranking. JEL-codes: C41, E32, J64. Gerard J. van den Berg acknowledges financial support of the Royal Netherlands Academy of Arts and Sciences. The authors wish to thank Jeffrey Smith and Sweder van Wijnbergen for helpful comments. 


\section{Introduction}

There are several ways to investigate the dynamics of unemployment. One way is to look at a specific point in time and consider the decomposition of unemployment into the product of incidence and expected duration of unemployment. A given stock of unemployed may originate from a range of combinations of incidence and duration, with at the one extreme a combination of small incidence and long duration and at the other extreme a combination of large incidence and short duration. Another way to investigate unemployment dynamics is to look at the behavior of unemployment and the related unemployment dynamics over the business cycle. Fluctuations in the number of unemployed may be caused by variations in incidence, average duration, or a combination of both. However, for a good understanding of the processes underlying unemployment dynamics, it is insufficient to restrict attention to a simple description of aggregate phenomena like fluctuations in the average unemployment duration, because there are competing explanations for this. One explanation is that individual unemployment duration distributions of all currently unemployed are varying over time. Alternatively, unemployment duration distributions may be constant at the individual level, in which case aggregate fluctuations are generated by variation in the composition of the inflow. Furthermore, the elapsed duration of the unemployment spell may reduce the probability of being hired by an employer. In that case there is so called negative duration dependence of the exit rate out of unemployment. Finally, there may be cyclical variation in the pattern of the negative duration dependence which is, as will be argued below, an indication of the persistence of unemployment.

In the past decades several elements of U.S. unemployment dynamics have been investigated empirically. The original focus of research was on the average duration of unemployment. Later on, other elements, like the cyclicality of incidence and duration, compositional effects, and duration dependence have been investigated.

The interest in average unemployment duration originates from a macroeconomic discussion about the functioning of the U.S. labor market. If unemployment is characterized by small incidence and long average duration then unemployment displays a high amount of persistence, which is interpreted as evidence of labor market malfunctioning. If unemployment durations are mostly short then they may be voluntary in nature and the result of rational behavior of unemployed for whom unemployment is a transitory and productive phase in their labor market career. The discussion boiled down to the issue whether unemploy- 
ment should be modelled in terms of stocks of persons who are unemployed for a long period of time, or as flows of persons who are unemployed for fairly short spells.

Empirical evidence in the discussion was usually based on information from the Current Population Survey (CPS). In most studies CPS information about elapsed unemployment durations at different points in (calendar) time was used to calculate expected completed durations of workers at the start of their unemployment spell. ${ }^{1}$

In the mid 1980s several CPS based studies focus on the relationship between unemployment dynamics and the business cycle. Sider (1985) examines the extent to which variations in unemployment reflect changes in the incidence of new spells or changes in average unemployment durations. According to Sider almost all cyclical unemployment resulted from changes in duration. Butler and McDonald (1986) estimate duration models separately for each calendar year and subsequently calculate an annual measure of inequality in the distribution of unemployment. From the correlation between this measure and business cycle indicators they conclude that during expansionary periods of the business cycle inequality in the distribution of unemployment decreases. ${ }^{2}$

Darby, Haltiwanger and Plant (1985) argue that systematic cyclical variation in the composition of the inflow into unemployment may cause cyclical fluctuations in aggregate unemployment, even if individual unemployment duration distributions do not change. In their theoretical model there are two groups of workers. The first group consists of job shoppers and is characterized by high rates of entry into and exit from unemployment. The second group consists of career makers who rarely become unemployed but need a lot of time to find a new job once they become unemployed. In booms, few career makers loose their jobs and most unemployment spells are related to job shopping. In recessions, however, relatively many career makers are fired, and job shopping is less frequent. Consequently, the average exit rate falls quickly when entering a recession, whereas the average exit rate increases slowly in a recovery from a recession.

\footnotetext{
${ }^{1}$ For examples, see Kaitz (1970), Clark and Summers (1979), Akerlof and Main (1980, 1981), and Carlson and Horrigan (1983). Since the end of the 1970s duration studies based on micro (panel and cross-section) data dominate in quantity over studies based on CPS data. The focus in these studies has been, amongst other things, on differences in exit behavior between groups of unemployed. See Devine and Kiefer (1991) for an overview.

${ }^{2}$ It should be pointed out that the steady-state assumption they make is not entirely consistent in the light of the fact that they estimate separate models for each calendar year. Moreover, their estimates of duration dependence are determined by the parametric functional form assumptions they make.
} 
Using CPS data they estimate time series regressions with the average exit probability from unemployment as dependent variable and the lagged share of short term unemployed, which they consider as an indicator of heterogeneity in the inflow, as one of the explanatory variables. They find that a high share of short term unemployed in a specific month increases the overall exit probability in the next month. From this they conclude that the changing composition is indeed an important determinant of cyclical unemployment fluctuations. Dynarski and Sheffrin (1990), who use data of the Panel Study of Income Dynamics, conclude however that changes in the composition in unemployment are not very important. Their conclusion is in line with Baker (1992a). Using CPS data, Baker shows that the variation in the composition of the inflow is insufficient to explain the variation in aggregate duration data, so "the heterogeneity explanation of aggregate variation sheds little light on the nature of unemployment dynamics'. Baker concludes that changes in individual unemployment durations are more important than compositional changes of the inflow. From ad hoc (graphical) checks, Imbens and Lynch (1992) obtain similar conclusions. A possible reason for the different results is that Baker, Dynarski and Sheffrin, and Imbens and Lynch use direct evidence of cyclical changes in the composition of the inflow whereas Darby et al. use an indicator variable which may reflect seasonal rather than cyclical effects.

On the issue of duration dependence there are also some studies based on CPS data (for an overview of the results from micro studies, see Devine and Kiefer (1991)). Butler and McDonald find positive duration dependence of the exit rate out of unemployment. Van den Berg and Van Ours (1996) analyze CPS time series of unemployment exit probabilities for different unemployment duration classes over the period 1967-91. They also find duration dependence.

It is not only important to establish whether or not there is negative duration dependence of the unemployment exit rate. It is also informative to establish whether there is a relationship between observed duration dependence and the business cycle. According to Blanchard (1991) and Blanchard and Diamond (1994) ranking by employers of unemployed workers according to the length of their unemployment spell may explain the existence of negative duration dependence. Ranking is particularly influencing exit rates of long term unemployed in a situation of labor market slack. In a recession applications by long term unemployed workers will be more frequently turned down for the reason that a short term unemployed worker has applied as well. Therefore, ranking causes duration dependence to be stronger during recessions and weaker during booms. Therefore, if there is ranking, exit rates of long term unemployed individuals are 
more sensitive to cyclical fluctuations than exit rates of short term unemployed individuals. There is some empirical evidence of differences in exit behavior between short and long term unemployed across the business cycle. According to Sider (1985), exit rates are more cyclically sensitive at shorter durations, i.e. less than 6 months. Butler and McDonald (1986) come to an opposite conclusion. According to them, increases in aggregate demand, as measured by real GNP, increase the exit rate most among those with longer durations of unemployment. Results from Dynarski and Sheffrin (1990) are in line with the study of Butler and McDonald. Dynarski and Sheffrin conclude that longer spells of unemployment are more sensitive to business cycle fluctuations (which they measure by the national unemployment rate).

All in all, a substantial amount of research on U.S. unemployment dynamics has been carried out in the past decades. There seems to be some sort of consensus about what is relevant in U.S. unemployment dynamics: on average unemployment durations are short, cyclical variation in unemployment is mainly caused by cyclical variation in the average unemployment duration and not by variation in incidence, changes in the composition of the inflow into unemployment are not very important, negative duration dependence of the exit rate out of unemployment is relevant, short term and long term unemployed react differently to business cycle fluctuations.

Some of the previous studies use quite simple techniques of analysis, are based on rather ad hoc assumptions or do not seem to have an entirely consistent approach. Yet, there has been an evolution in research technology moving from analyses that depended heavily on steady state assumptions to more complex models of unemployment dynamics allowing for a non-steady state environment. However, it is also clear that researchers have focused on separate elements of unemployment dynamics. What lacks is a study that takes simultaneously all elements of unemployment dynamics into account.

This paper is an attempt to fill this gap. We give a complete empirical overview of U.S. unemployment dynamics. We investigate the relevance of traditional components like incidence, duration, inflow composition and the effects of the business cycle. We also investigate the phenomenon of ranking, which causes negative duration dependence.

When investigating the nature of the decline of individual exit rates over the duration of unemployment the researcher has to take the phenomenon of unobserved heterogeneity into account. Unobserved heterogeneity causes the observed exit rate of an apparantly homogeneous group of unemployed to decline over the duration of unemployment, because the best qualified unemployed leave 
unemployment first. The sorting caused by heterogeneity has other cyclical implications than ranking. In the top of the cycle the exit rate declines very rapidly. In slumps even the best qualified unemployed find it difficult to leave unemployment, and the aggregate exit rate declines only mildly over the duration of unemployment. So, the sorting model implies that duration dependence is less negative in a recession than at the top of the business cycle. Because of the difference in cyclical behavior, the pattern of interaction between duration and cycle is informative on the dominance of ranking and sorting effects. ${ }^{3}$

The outline of the paper is as follows. Section 2 contains the theoretical part of the paper. Subsection 2.1 discusses sorting, ranking and duration dependence more extensively. In Subsection 2.2 a stylized theoretical model of ranking and sorting is discussed. In Subsection 2.3 we develop an empirical duration model that allows for interaction between aggregate duration dependence and the business cycle. The parameterization of this model is given in Subsection 2.4. Section 3 discusses the data set. We use aggregate CPS data on unemployment classified by unemployment duration and a limited number of demographic characteristics. Section 4 presents parameter estimates of our model. Subsection 4.1 summarizes the main results. Subsection 4.2 discusses robustness of our results with respect to the introduction of cyclical variation in the composition of the inflow. In Section 5, we use the parameter estimates to evaluate the role of various effects in explaining aggregate fluctuations in unemployment. Subsection 5.1 discusses an elementary decomposition in incidence and duration, whereas 5.2 evaluates duration dependence and interaction effects. Section 6 concludes.

Three appendices are added to complement the main text with details. Appendix A derives the properties of the theoretical sorting model in Subsection 2.2. Appendix B provides details on the decomposition of Section 5. We discuss identification in Appendix C.

\section{A flexible empirical model of the dynamics in unemployment durations}

\subsection{Ranking and sorting}

It is a well established fact that long term unemployed have on average lower exit rates from unemployment than short term unemployed. In the U.S., according

\footnotetext{
${ }^{3}$ Note that we do not distinguish between the effects of sorting and ranking because we are interested in sorting per se, but because we have to account for it in order to be able to investigate the importance of ranking.
} 
to CPS data the aggregate exit probability falls from over $50 \%$ per month in the first month of unemployment to around $15 \%$ per month in the fifth month.

Observed negative duration dependence could be caused by negative duration dependence at the micro level. In a world with asymmetric information, high unemployment durations may signal low worker quality, and direct links between unemployment duration and worker productivity may exist if skills deteriorate when unemployed. In either case, employers prefer to hire short term unemployed over hiring long term unemployed. Consequently, short term unemployed have higher exit rates into employment.

Blanchard (1991) discusses ranking as an explanation of negative duration dependence at the individual level. Ranking reflects employers' recruiting behavior. Employers prefer to hire short term unemployed workers over long term unemployed workers, because of either the signalling effect or the loss of skills mentioned earlier. Ranking may even occur if there is only a slight deterioration of human capital, because the employer still prefers a short term unemployed to a long term unemployed since the latter has a disadvantage, be it a small one.

As argued in Section 1, sorting also causes negative duration dependence of observed exit probabilities. A crucial difference between ranking and sorting is that ranking is caused by variation in elapsed unemployment durations, whereas sorting is driven by variation in innate characteristics, like ability, and characteristics that can be considered given in the short run, like education. As a result, ranking and sorting have different dynamic implications. In a pure sorting model, unemployment exit rates of short term unemployed are more sensitive to cyclical fluctuations than exit rates of long term unemployed (Van den Berg and Van Ours $(1994,1996,1997))$. In a pure ranking model, exit rates of long term unemployed are more sensitive to cyclical fluctuations than exit rates of short term unemployed (Blanchard (1991) and Blanchard and Diamond (1994)). By studying the interaction between cyclical fluctuations and exit rates at different unemployment durations we can distuinguish between ranking and sorting.

This distinction is important as ranking and sorting have different allocative and macroeconomic implications. In case of pure sorting, long term unemployment is concentrated in certain segments of the labor market. This may be undesirable and call for government intervention targeted at these specific segments. Pure ranking, on the other hand, is not necessarily discriminative between segments of the labor market. Instead, it is a future risk borne equally by all newly unemployed at each point in time. It could, however, be related to real economic costs of long term unemployment. If ranking is driven by loss of skills during unemployment, prolonged spells of unemployment lead to excessive loss of human 
capital. Similarly, even if ranking is based on a mild loss of skills it could induce demotivation among long term unemployed. In either case, the government may want to direct policy to prevent the emergence of long term unemployment.

The difference in macroeconomic implications of both models is related to the allocative differences. In case of ranking, newly unemployed face better employment prospects than the currently unemployed. Thus, in labor markets where the currently employed bargain over wages with employers, the presence of long term unemployed has little effect on wages. Consequently, even if ranking is based on a mild loss of skills it may cause demotivation of long term unemployed and thus considerable persistence in unemployment (Blanchard (1991)). In a sorting model, however, the presence of long term unemployed can be expected to weaken the effect of the level of unemployment on wages, just like overall unemployment does (Blanchard and Diamond (1994)). As, in this case, prolonged unemployment does not necessarily weaken the effect of unemployment on wages, sorting is less likely to be associated with unemployment persistence.

One may argue that, within markets, employers rank on both unemployment duration and skills. In that case, ranking is not rationalized by loss of skills, but by the fact that long term unemployed are more likely to be screened and rejected by other employers in the same market. Formalization of this idea, however, is beyond the scope of this paper, and will be left for future research.

\section{$2.2 \quad$ A formal framework}

In this subsection we provide a model framework that incorporates both ranking and sorting. We do not claim that this model provides a perfect description of the labor market. Rather, it provides a consistent description of a labor market in which ranking and sorting can be distinguished. As such, it serves as a benchmark model later on, when we discuss the empirical operationalization of these concepts. It should be stressed from the outset that this formal model is not imposed on the data.

Sorting occurs if there is variation across individuals in the level of the exit rate from unemployment. The main assumption of this subsection is that all of this variation can be traced back to labor market segmentation. We assume that the labor market is segmented in, possibly uncountably many, unrelated submarkets or segments and that each individual searches in only one segment. Exit rates from unemployment may vary between the submarkets, but within segments each individual with the same unemployment duration has the same exit rate to employment. Clearly, both from the perspective of supply and demand 
this assumption can be criticized. For example, it seems that, in practice, there is no supply segmentation of the labor market in submarkets for able and less able people, although ability may be related to the exit rate to employment. On the demand side, one could argue, workers from various segments may, for instance, be complements in the production process, which implies that hiring decisions in the various segments may be related. Our full segmentation assumption does not allow for such interaction between submarkets. On the other hand, it is not unreasonable to assume that segments exist for, for example, schooling, as, certainly in the short run, individuals cannot move from one schooling segment to the other. Furthermore, the segmentation assumption greatly facilitates the introduction of ranking into the model framework. Ranking occurs if, for whatever reason, firms prefer to hire short term unemployed. Due to the full segmentation and homogeneity within segments, the Blanchard and Diamond (1994) model is directly applicable to each of the submarkets. Thus, we have ranking within segments, and sorting between segments. ${ }^{4}$

Suppose that unemployment duration is measured on a discrete time scale with origin 0 , and denote the elapsed duration of a spell of unemployment for a given individual by $t$. Suppose that segments can be characterized by a scalar $v \in(0, \infty)$. The probability of an individual leaving unemployment in segment $v$ at unemployment duration $t$, conditional on survival up to duration $t$ and state of the business cycle $c$, is given by

$$
\vartheta(t \mid c, v)=\eta(c, t) v
$$

The individual exit probability is proportional in $\eta(t, c)$, which represents business cycle and duration dependence of individual exit probabilities, and a segment specific effect $v$. Note that $\eta(c, t)$ is common to all individuals within a segment $v$. The distribution of individuals over the segments $v$ reflects all heterogeneity across unemployment durations, apart from random variation in these durations. We denote the distribution function of unemployed over the segments $v$ by $G(v)$. As $\vartheta(t \mid c, v)$ is a probability, $G(v)$ is required to satisfy $\operatorname{Pr}[0<\vartheta(t \mid c, v)<1]=$ 1. Suppose that $\eta(c, t)$ is differentiable with respect to $c$, where $\eta_{c}(c, t)>0$ : individual exit probabilities are higher at higher values of the business cycle indicator. If employers do not rank applicants according to $t$, but hire randomly, individual exit probabilities do not change with duration: $\eta(c, t+1)=\eta(c, t)$

\footnotetext{
${ }^{4}$ Note that the previous empirical literature on unemployment dynamics does not make segmentation assumptions. This is because this literature is not concerned with ranking, which is based on employers' behavior and therefore needs assumptions on market structure. Our empirical model does however contain the models in this literature as special cases.
} 
and $\partial \log [\eta(c, t+1) / \eta(c, t)] / \partial c=0 .^{5}$ Ranking, however, implies that individual exit probabilities decrease during unemployment; $\eta(c, t+1)<\eta(c, t)$. Moreover, within segments the labor market is homogeneous, so it follows from Blanchard (1991) and Blanchard and Diamond (1994) that in case of ranking:

$$
\frac{\partial \log [\eta(c, t+1) / \eta(c, t)]}{\partial c}>0
$$

Thus, duration dependence caused by ranking is less steep in a boom, when $c$ is relatively large. In other words, exit probabilities from unemployment are more sensitive to business cycle fluctuations at higher durations. These are the two faces of what we label to be positive interaction. The intuition behind this has been discussed in Section 1 and Subsection 2.1.

Now consider the effect of sorting. The aggregate exit probability in a cohort of workers that has become unemployed some time $t$ ago, in a state of the business cycle $c$, equals ${ }^{6}$

$$
\vartheta(t \mid c)=\eta(c, t) \nu(c, t)
$$

where $\nu(c, t):=\mathbb{E}[v \mid c, \mathbf{t} \geq t]=\mathbb{E}\left[v(1-\eta(c) v)^{t}\right] / \mathbb{E}\left[(1-\eta(c) v)^{t}\right]$. Note that $\nu(c, 0)=\mathbb{E}[v]$. If the distribution of $v$ is degenerate, i.e. if all workers are concentrated in a single segment, $\nu(c, t)=\mathbb{E}[v], \nu_{c}(c, t)=0$ and $\nu(c, t+1)=$ $\nu(c, t)$. In this case, there is no sorting, as workers are homogeneous. Now suppose that $\operatorname{var}(v)>0$. Then, workers are heterogeneous, and sorting will occur and cause negative duration dependence at the aggregate level: $\nu(c, t+1)<\nu(c, t)$. In Appendix $\mathrm{A}$ it is shown that sorting implies that duration dependence between durations 0 and $t$ is steeper in booms: $\partial \log [\nu(c, t) / \nu(c, 0)] / \partial c<0$ for any $t>0$. As, by implication of (2), in case of ranking

$$
\frac{\partial}{\partial c} \log \frac{\eta(c, t)}{\eta(c, 0)}=\sum_{i=1}^{t} \frac{\partial}{\partial c} \log \frac{\eta(c, i)}{\eta(c, i-1)}>0
$$

a test can be constructed from interaction between the business cycle and the level of duration dependence between duration 0 and any duration $t>0$. We will label this the cumulative interaction effect. A measure of the overall cumulative

\footnotetext{
${ }^{5}$ Note that in this subsection, at this stage, we do not yet consider other reasons for duration dependence, like a decreasing search intensity.

${ }^{6}$ We use bold fonts for random variables and standard fonts for their realizations, only if there is a risk of confusion. Appendix A provides details. In Abbring, Van den Berg and Van Ours (1997) we show that qualitatively similar results hold in the continuous time Mixed Proportional Hazard version of our model.
} 
interaction effect in our combined ranking-sorting labor market is found by taking the derivative with respect to $c$ of the $\log$ exit probability at time $t$, relative to the exit probability at time 0 , as in

$$
\frac{\partial}{\partial c} \log \frac{\vartheta(t \mid c)}{\vartheta(0 \mid c)}=\frac{\partial}{\partial c} \log \frac{\eta(c, t)}{\eta(c, 0)}+\frac{\partial}{\partial c} \log \frac{\nu(c, t)}{\nu(c, 0)}
$$

The sign of the overall cumulative interaction effect is determined by the relative importance of ranking and sorting. So, a test on ranking versus sorting can be constructed from interaction data by evaluating (5) at various durations, testing for the sign of the interaction effect at each duration. Clearly, both sorting and ranking may occur simultaneously in the data. Then, the test merely gives the dominant process up to each unemployment duration. If the test statistic is negative for small $t$ and positive for large $t$ then both sorting and ranking occur.

If we abandon our stylized model of ranking and sorting, theoretical results become less clear cut. Lebon (1993) shows that, in a labor market with loss of skills during unemployment, firms are likely to rank in booms, but randomly hire in recessions. Blanchard (1996) focuses on the effect of wage differentials between short and long term unemployed, and concludes the opposite. In our empirical model which we present in the next section we account for this by allowing for asymmetries in interaction effects over the business cycle. Moreover, we allow for other types of duration dependence.

\subsection{Empirical model specification}

In this subsection we present a flexible model for the empirical analysis of the dynamics in the unemployment duration distribution. This model is estimable with CPS data. We will show that it captures the phenomena described in the previous subsections and that it is well-suited to study the other issues discussed in the literature.

We distinguish two discrete measures of time, unemployment duration and calendar time, with the same measurement scales, but different origins. The duration of a spell of unemployment for a given individual is denoted by $t$ and has time origin 0 . Calendar time is denoted by $\tau$ and has time origin $\tau_{0}$. Furthermore, we distinguish demographic groups of workers, indexed by $g$, and denote the exit probability from unemployment of a cohort of workers from group $g$ after $t$ periods of unemployment, given that they have been unemployed for $t$ periods at calendar time $\tau$ by $\theta_{g}(t \mid \tau)$. We can calculate these exit probabilities using information on the number of individuals in group $g$ that have been unemployed for $t$ periods of time at calendar time $\tau\left(U_{g}(t \mid \tau)\right)$ and the number of these individuals that have 
been unemployed for $t+1$ periods of time at calendar time $\tau+1\left(U_{g}(t+1 \mid \tau+1) \leq\right.$ $\left.U_{g}(t \mid \tau)\right)$, as

$$
\theta_{g}(t \mid \tau)=\frac{U_{g}(t \mid \tau)-U_{g}(t+1 \mid \tau+1)}{U_{g}(t \mid \tau)} .
$$

In reality, we do not exactly observe the numbers $U_{g}(t \mid \tau)$. The CPS data we use are based on surveys of unemployed individuals. Therefore the data contain sampling errors. Furthermore, respondents may have difficulties recalling their elapsed unemployment durations. In that case, they may be counted as being unemployed for $t$ periods of time whereas in reality they are unemployed for $t-1$ or $t+1$ periods. Finally, respondents may tend to round off their duration to the nearest natural unit of time like an integer number of months (see also Section 3). Deviation of unemployment figures from their true values causes deviation of observed exit probabilities from their true values, and may even render these probabilities negative or larger than 1 . Therefore, we will allow for measurement errors in the model. From now on we place a tilde on top of observed values, in contrast to true, or unobserved, values. We assume that

$$
\widetilde{U_{g}}(t \mid \tau)=U_{g}(t \mid \tau) \varepsilon_{g, t, \tau}
$$

with

$$
\log \varepsilon_{g, t, \tau} \sim N\left(0, \sigma_{g}^{2}\right)
$$

and independence between the error terms. Note that we allow for heteroskedasticity between demographic groups. From this specification, it follows that the observed $\log \left(1-\tilde{\theta}_{g}(t \mid \tau)\right)$ equals the sum of the true $\log \left(1-\theta_{g}(t \mid \tau)\right)$ and a disturbance term. Thus, if we specify a model for the true hazard rates $\theta_{g}$, we can express the observed hazard rates in terms of the 'parameters' and a random disturbance term, and the model parameters can be estimated.

We assume that all variation in the true exit probabilities out of unemployment can be explained by demographic group $g$, the prevailing unemployment duration $t$, calendar time $\tau$, and a general interaction effect of calendar time and duration dependence. We use a multiplicative specification of the exit probability out of unemployment with functions that represent these effects as arguments: ${ }^{7}$

$$
\theta_{g}(t \mid \tau)=\exp \left(\alpha_{0, g}\right) \psi_{1}(t) \psi_{2}(\tau) \psi_{3}(t, \tau)
$$

\footnotetext{
${ }^{7}$ In terms of the notation of Subsection 2.2 we have that $\theta_{g}(t \mid \tau)$ is equivalent to $\vartheta(t \mid c)$ for given demographic characteristics. A state $c$ of the business cycle corresponds to a time interval within which $\psi_{2}(\tau)$ is constant.
} 
Observed demographic characteristics are only allowed to affect the constant term in the exit probability. The function $\psi_{1}(t)$ captures types of individual duration dependence that have not been discussed in the previous subsection, like a decreasing search intensity due to a discouraged worker effect, and limited benefit entitlement. ${ }^{8}$ The calendar time function $\psi_{2}(\tau)$ is specified as the product of a seasonal term $\omega_{2}(\tau)$, and a cyclical and trend term $\psi_{2, c}(\tau)$ :

$$
\psi_{2}(\tau)=\omega_{2}(\tau) \psi_{2, c}(\tau)
$$

The general interaction effect is decomposed in seasonal and cyclical effects depending on the moment of inflow, $\omega_{3}(\tau-t)$ and $\psi_{3, c}(\tau-t)$, and a specific interaction effect $\psi_{3, i}(t, \tau)$ that represents the interaction effects that are caused by ranking and sorting: ${ }^{9}$

$$
\psi_{3}(t, \tau)=\omega_{3}(\tau-t) \psi_{3, c}(\tau-t) \psi_{3, i}(t, \tau)
$$

We restrict the functional form of $\psi_{3, i}(t, \tau)$ to satisfy $\psi_{3, i}(\bar{t}, \tau)=\psi_{3, i}(t, \bar{\tau})=1$, for some given $\bar{t}$ and $\bar{\tau}$. With the normalization imposed, $\psi_{2, c}(\tau)$ can be seen as the 'baseline' trend and cycle experienced by unemployed in duration class $\bar{t}$, and $\psi_{1}(t)$ is the 'baseline' duration dependence experienced at calendar time moment $\bar{\tau}$. We identify business cycle fluctuations as fluctuations in $\psi_{2, c}(\tau)$. Then sorting implies that, for $t>0, \psi_{3, i}(\tau, t) / \psi_{3, i}(\tau, 0)$ is smaller for $\tau$ for which $\psi_{2, c}(\tau)$ is higher, i.e. that duration dependence is more negative in booms. In case of ranking, the opposite holds.

\footnotetext{
${ }^{8}$ In Subsection 2.2 we only considered duration dependence as a result of ranking and, on an aggregate level, as a result of sorting. To the extent in which other types of individual duration dependence matter for employers, the inclusion of these would complicate the theoretical model, since it is likely that employer behavior towards ranking would be affected. Here we simply assume that these other types of duration dependence can be represented by way of a multiplicative term in the individual exit probabilities. Thus, we also assume that such duration dependence does not vary over the business cycle. Without the latter, the interaction effect would be affected by the way in which this duration dependence varies over the business cycle, and as a result it would not be informative on ranking and sorting.

${ }^{9}$ Note that the theoretical framework of Subsection 2.2 does not take account of functions $\omega_{3}(\tau-t)$ and $\psi_{3, c}(\tau-t)$. In the main empirical analysis we do not allow for $\psi_{3, c}(\tau-t)$ either. We simply include the seasonal effect $\omega_{3}(\tau-t)$ in the empirical specification for reasons of flexibility. In Subsection 4.2 below we argue that the functions $\omega_{3}$ and $\psi_{3, c}$ can be used to a certain extent to capture the way in which the composition of the inflow changes over time in the data. In terminology of Subsection 2.2, a changing composition of the inflow means that the inflow of workers attached to a certain segment $v$ varies as a fraction of the total inflow. It is clear that the functions $\omega_{3}$ and $\psi_{3, c}$ are not identified without additional restrictions. We discuss this in detail in the appendix and in Subsection 4.2.
} 
The interaction between the business cycle and duration dependence is, in its most general form, specified as

$$
\psi_{3, i}(t, \tau)=\exp \left[\xi_{1}(t) \xi_{2}(\tau)\right]
$$

with $\xi_{1}(\bar{t})=\xi_{2}(\bar{\tau})=0$. This is an extremely general specification that allows the interaction sign to vary with duration as well as with the state of the business cycle. We clarify this specification by considering two special cases in which we abstract from seasonal and cyclical fluctuations in the composition of the inflow so that equation (9) becomes: $\theta_{g}(t \mid \tau)=\exp \left(\alpha_{0, g}\right) \psi_{1}(t) \psi_{2}(\tau) \exp \left[\xi_{1}(t) \xi_{2}(\tau)\right]$. In the special cases we impose restrictions on either the duration part or the calendar time part of the interaction effect:

i) restriction on the duration part of the interaction effect

Suppose that $\xi_{1}(t)=\log [(t+1) /(\bar{t}+1)]$, which implies that the interaction has the same sign in all duration classes. Overall duration dependence is now given by

$$
\log \frac{\theta_{g}(t \mid \tau)}{\theta_{g}(0 \mid \tau)}=\log \frac{\psi_{1}(t)}{\psi_{1}(0)}+\xi_{2}(\tau) \log (t+1)
$$

Duration dependence between duration classes 0 and $t$ is more negative if $\xi_{2}(\tau)$ is smaller. Thus, if $\psi_{2, c}(\tau)$ and $\xi_{2}(\tau)$ are positively correlated, we have positive interaction, duration dependence is more positive in booms, and the ranking effect dominates. If $\psi_{2, c}(\tau)$ and $\xi_{2}(\tau)$ are negatively correlated the sorting effect dominates. Similarly, the relative state of the cycle in duration class $t$ is given by

$$
\log \frac{\theta_{g}(t \mid \tau)}{\theta_{g}(t \mid \bar{\tau})}=\log \frac{\psi_{2}(\tau)}{\psi_{2}(\bar{\tau})}+\xi_{2}(\tau) \log \frac{(t+1)}{(\bar{t}+1)}
$$

In case of positive interaction, cycles are amplified at higher durations, in case of negative interaction cycles are dampened at higher durations.

A specification with a flexible function $\xi_{2}(\tau)$ allows for asymmetries in the interaction effect over the business cycle. In this example, where the interaction effect has the same sign at each duration at a given point time, this means that the interaction effect may switch sign in the course of calendar time. Thus, ranking effects may dominate at some point in time, whereas sorting effects may dominate at another moment. According to Blanchard (1991), this may be a relevant description of unemployment dynamics in case of severe skill deterioration or severe worker discouragement at high durations. In a recession, durations are often 
high, and many workers will suffer such irreversible changes in their skills or taste.

ii) restriction on the calendar time part of the interaction effect

Suppose that $\xi_{2}(\tau)=\log \left[\psi_{2}(\tau) / \psi_{2}(\bar{\tau})\right]$ that is, the calendar time effect in the interaction term is the same as the calendar time effect in the basic model. Overall duration dependence and the relative state of the cycle are now given by

$$
\log \frac{\theta_{g}(t \mid \tau)}{\theta_{g}(0 \mid \tau)}=\log \frac{\psi_{1}(t)}{\psi_{1}(0)}+\left[\xi_{1}(t)-\xi_{1}(0)\right] \log \frac{\psi_{2}(\tau)}{\psi_{2}(\bar{\tau})},
$$

and

$$
\log \frac{\theta_{g}(t \mid \tau)}{\theta_{g}(t \mid \bar{\tau})}=\left[1+\xi_{1}(t)\right] \log \frac{\psi_{2}(\tau)}{\psi_{2}(\bar{\tau})} .
$$

In this case, interaction effects may have different signs at different duration classes, and cyclical sensitivity may depend non-monotonously on duration. However, asymmetries of interaction effects over the cycle are excluded by this specification.

The most flexible specification of the interaction effect, as in equation (12), combines both features, by allowing for both asymmetries over duration and asymmetries over the business cycle.

The functions $\omega_{3}(\tau-t)$ and $\psi_{3, c}(\tau-t)$ can be thought to represent seasonal and cyclical fluctuations in the composition of the inflow, where composition refers to composition in terms of characteristics that are related to exit probabilities. As these functions introduce a second type of interaction between duration and calendar time in our model, the question arises which assumptions have to be made to identify these interaction effects. In Appendix $\mathrm{C}$ we discuss identification of our interaction model.

\subsection{Parameterization}

The baseline duration dependence function is fully flexible since it is parameterized as a step function which has the same value within duration intervals but may differ between duration intervals:

$$
\psi_{1}(t)=\exp \left\{\sum_{i=0}^{n_{t}-1} \psi_{1 i} I_{1, i}(t)\right\},
$$

in which $I_{1, i}(t)=1$ if $t=i$ and 0 otherwise, and $n_{t}$ is the number of duration classes considered. The seasonal effects are specified by

$$
\omega_{2}(\tau)=\exp \left\{\sum_{s=1}^{S} \omega_{2 s} I_{s}(\tau)\right\},
$$


where $I_{s}$ is an indicator function for season $s, s=1, \ldots, S$, and similarly for $\omega_{3}(\tau-t)$. Cyclical and trend effects are represented by a flexible polynomial of degree $n$. We could parameterize this polynomial in the standard way, and specify it as being composed of polynomial terms $\alpha_{2 i} \tau^{i}, i=0, \ldots, n$. However, as the terms $\tau^{i}$ are not mutually orthogonal, iterative estimation of the parameters $\alpha_{2 i}$ may be slow because of multicollinearity problems. To avoid these problems, we use Chebyshev polynomials of the first kind. Thus, we do not construct the polynomial from terms $\alpha_{2 i} \tau^{i}$, but from terms $\alpha_{2 i} p_{i}(\tau), i=0, \ldots, n$, where $p_{0}(\tau), p_{1}(\tau), \ldots, p_{n}(\tau)$ are mutually orthogonal polynomials of indexed degree. ${ }^{10}$ Then, the baseline trend and cycle $\psi_{2, c}(\tau)$ is specified as

$$
\psi_{2, c}(\tau)=\exp \left\{\sum_{i=0}^{n} \alpha_{2 i} p_{i}(\tau)\right\} .
$$

We will also try an alternative specification of $\psi_{2, c}(\tau)$, in which we specify $\psi_{2, c}(\tau)=$ $\exp \left(\alpha_{20}+\alpha_{21} p_{1}(\hat{\tau})+\beta_{2} c(\tau)\right)$, where $c(\tau)$ is an observed indicator of the business cycle, in particular a help wanted index.

As indicated in the previous subsection, in our baseline analysis, the effect on the composition of the inflow is restricted to seasonal fluctuations.

We specify the duration part of the interaction effect, $\xi_{1}(t)$, either by $\log (t+1)$, or by

$$
\xi_{1}(t)=\xi_{11}^{*} t+\xi_{12}^{*} t^{2}
$$

The first specification has already appeared in example (i) of Subsection 2.3. As $\log (t+1)$ is increasing at all $t$, this specification implies that the interaction effect has the same sign at all durations. If $\xi_{2}(\tau)$ is tracking the cycle in the outflow, duration dependence will be less negative at all durations in the top of a cycle. If $\xi_{2}(\tau)$ is flexibly specified, it could be procyclical in one and countercyclical in an other period. Then, the interaction effect switches sign over calendar time. This is what we have labeled 'asymmetry over calendar time' earlier.

The quadratic specification (20) is more flexible, as it allows the interaction effect to switch sign once over duration. For example, if $\xi_{1}(t)$ is U-shaped and

\footnotetext{
${ }^{10}$ More specifically, we first linearly transform the calendar time domain to the domain of orthogonality of the Chebyshev polynomial, $[-1,1]$, by means of

$\hat{\tau}(\tau)=2 \frac{\tau-\tau_{0}}{n_{\tau}-1}-1$,

where $n_{\tau}$ is the number of calendar time periods considered. The series of orthogonal polynomials is then generated by (see Abramowitz and Stegun (1970), Table 22.3)

$p_{0}(\tau)=1$, and

$p_{k}(\hat{\tau})=\frac{k}{2} \sum_{i=0}^{\left[\frac{k}{2}\right]}(-1)^{i} \frac{(k-i-1) !}{i !(k-2 i) !}(2 \hat{\tau})^{k-2 i}$ for $i=1,2, \ldots, n$.
} 
$\xi_{2}(\tau)$ is procyclical, in booms duration dependence between consecutive duration classes will be more negative at low durations, and more positive at high durations. This is what we have called 'asymmetry over duration' earlier. From Subsection 2.2 we know that positive interaction at high durations can be induced by both ranking and sorting. An unambiguous test can only be constructed from the cumulative interaction effect, i.e. the variation of duration dependence between durations 0 and $t$ over the cycle. Therefore, we will not only report estimates of $\xi_{10}:=\xi_{11}^{*}+\xi_{12}^{*}$ and $\xi_{11}:=2 \xi_{12}^{*}$, but also report the resulting estimates of $\xi_{1}(t)$. $\xi_{1}(t+1)-\xi_{1}(t)=\xi_{10}+\xi_{11} t$ can be shown to give the interaction effect between duration classes $t$ and $t+1$ if $\xi_{2}(\tau)=\log \psi_{2, c}(\tau)$. As can be inferred from example (ii) of Subsection 2.3, $\xi_{1}(t)$ gives the cumulative interaction effect in this case. If $\xi_{2}(\tau)$ is flexibly specified, the sign of the cumulative interaction effect is the sign of $\xi_{1}(t)$ if $\xi_{2}(\tau)$ is procyclical, and minus the sign of $\xi_{1}(t)$ otherwise.

We specify the calendar time part of the interaction effect, $\xi_{2}(\tau)$, by $\xi_{2}(\tau)=$ $\log \psi_{2, c}(\tau)$, or by

$$
\xi_{2}(\tau)=\exp \left\{\sum_{i=0}^{n} \alpha_{3 i} p_{i}(\tau)\right\} .
$$

As is clear by now, the first specification prevents the sign of the interaction effect to switch over calendar time. As $\xi_{2}(\tau)$ is, by construction, procyclical in this case, the sign of $\xi_{1}(t)$ can now directly be read as the sign of the cumulative interaction effect. If we use specification (21) instead, interaction effects are allowed to change sign over calendar time. This is again asymmetry over calendar time. We have to check the nature of the cyclicality of $\xi_{2}(\tau)$ before we can judge the interaction effect implied by $\xi_{1}(t)$. Clearly, if $\xi_{2}(\tau)$ is procyclical, the interaction effect has the same sign as $\xi_{1}(t)$. Otherwise, it has the opposite sign.

For the seasonal effects we use 4 quarterly indicators. Both $\psi_{2, c}(\tau)$ and, if specified according to $(21), \xi_{2}(\tau)$ are specified with $n=15 .^{11}$

\section{Data}

To estimate our model we use the same data as Van den Berg and Van Ours (1996). These data are based on unpublished CPS data from the U.S. Department

\footnotetext{
${ }^{11}$ Furthermore, we normalize $\omega_{21}=\omega_{31}=0$. Apart from that, we normalize $\psi_{1}(0)=1 \Leftrightarrow$ $\psi_{10}=0, \alpha_{20}=\sum_{i=1}^{[n / 2]}(-1)^{i+1} \alpha_{2,2 \cdot i}$, and $\alpha_{30}=\sum_{i=1}^{[n / 2]}(-1)^{i+1} \alpha_{3,2 \cdot i}$. The last 2 normalizations ensure that $\psi_{2, c}(\tau)$ and $\psi_{3, c}(t, \tau)$ equal 1 in the sample mean calendar moment, $\hat{\tau}^{-1}(0)$. Similarly, we take the help wanted index in log deviations from its value in the mean calendar time moment. Finally, note that we have implicitly chosen $\bar{t}=0$ and $\bar{\tau}=\hat{\tau}^{-1}(0)$.
} 
of Labor, which give monthly information on unemployment by weekly duration classes. In the analysis we use time series of monthly unemployment figures for four groups of workers, white males, white females, black males and black females, over the period January 1968-May 1992. Thus, we observe changes in the race and gender composition of the inflow. Since the information on exit rates becomes more unreliable at longer durations we only use information on exit rates for the first six months of unemployment.

As, for example, Sider (1985) and Baker (1992a) pointed out, there are several problems connected to the use of these data. First of all, the way in which the data are collected implies that we cannot follow actual cohorts over time. However, we may consider the data as synthetic cohorts. Second, the empirical analysis is facilitated if the frequency at which the data are collected equals the sizes of the unemployment duration classes. We aggregate the weekly duration classes into monthly duration classes. Finally, the data are influenced by phenomena like digit preferences and the tendency of respondents to report 'weeks of unemployment' as whole months (Baker (1992b)). Because of this we made the same corrections as in Baker (1992a). Baker reallocated 30 percent of the respondents at 4, 8, 12, 16 and 26 weeks, 40 percent of those at 52 weeks, and 50 percent of those at 78 and 99 weeks, in each month of the sample to adjacent later weeks.

The data do not enable us to make a distinction between employment and transition out of the labor force as alternative destinations. But, as Abowd and Zellner (1985) show, on average, the share of workers becoming employed in the outflow from unemployment is larger than the share of workers leaving the labor force.

Finally, we use the help wanted index from Citibase as a cyclical indicator $c(\tau)$. Data on this indicator are plotted in Figure 1.

\section{Estimation results}

\subsection{Parameter estimates}

Table 1 contains estimates of four different specifications of our baseline model. The first column contains estimates of the most flexible model, where $\xi_{1}(t)$ is assumed to have a quadratic specification. The second and third column show estimates of models in which we restricted $\xi_{1}(t)=\log (t+1)$ and $\xi_{2}(\tau)=\log \psi_{2, c}(\tau)$, respectively. In the last column, the flexible specification of $\log \psi_{2, c}(\tau)$ as a polynomial is abandoned, and replaced by a more restrictive, but also more comprehensible combination of a linear trend and our business cycle indicator, the help 
wanted index.

Comparing the estimation results of Columns 2-4 with those of Column 1 we draw two conclusions. The first conclusion is that many parameter estimates are hardly affected by the restrictions imposed. So, the estimated coefficients are quite robust. The second conclusion, however, is that the restrictions do affect the fit of the model to the data. This follows from likelihood ratio tests at conventional levels of significance. Therefore, we restrict the discussion of the estimation results to the estimates presented in Column 1 of Table 1.

The differences between the four $\alpha_{0}$-coefficients reflect the differences in exit probabilities between the different groups of unemployed workers. Conditional on the state of the business cycle, the season and the duration of unemployment white female unemployed have the highest exit probability out of unemployment while black male unemployed have the lowest exit probability. There is obviously a bigger difference between males and females than there is between black and white unemployed. The estimates of the $\alpha_{2}$-coefficients indicate that there is calendar time fluctuation in the individual exit probability out of unemployment.

The estimates of $\psi_{1}(t)$ imply significant negative duration dependence at all duration classes. As the parameter values of the polynomial are not very informative on the shape of $\log \psi_{2, c}(\tau)$, we have plotted this function in Figure 2. As explained earlier, $\log \psi_{2, c}(\tau)$ corresponds to the cycle at $t=\bar{t}=0$. Comparison with the help wanted index in Figure 1 indicates that exit probabilities are procyclical. This is confirmed by the parameter on the help wanted index in the fourth estimate, which is significantly positive. So, in upswings of the economy unemployment durations get shorter, in downswings they get longer.

The $\omega_{2}$-coefficients indicate to what extent there is seasonal fluctuation in the exit probability. It appears that there are seasonal fluctuations of this kind, the exit probability in the third quarter being the highest and in the first quarter being the lowest. Labor market conditions seem to be most favorable in the summer months. The estimated $\omega_{3}$-coefficients indicate that there is also seasonal variation in the exit probability caused by seasonal variation in the quality of the inflow conditional on the other determinants of the unemployment exit probability. Unemployed who enter in the third quarter have the lowest exit probability while those entering in the first quarter have the highest exit probability. The reason for this might be that the incidence is largest in the third quarter and smallest in the first quarter. So, unemployed entering in the third quarter face a lot of competition, while for those entering the first quarter competition is less severe.

Interaction effects are generally significant. Interaction is significantly nega- 
tive in the first month, and significantly positive in later months. Figure 3 plots $\log$ cycles and duration dependence $\left(\log \left[\psi_{1}(t) \psi_{2, c}(\tau) \psi_{3, i}(t, \tau)\right]\right)$ in the exit probabilities by duration class. Cyclical sensitivity falls between duration classes 0 and 1 , but rises from $t=1$ onwards. In duration classes 3 and 4 , cyclical sensitivity is higher than in duration class 0 . Figure 4 offers a different angle by plotting duration dependence in $\log$ exit probabilities at $\xi_{2}(\tau)=0.20$ and $\xi_{2}(\tau)=-0.20$, which roughly correspond to a boom and a recession period. In a boom, duration dependence is relatively high in the first month, but relatively low in the later months. We conclude that sorting is the dominant process in the first two months of unemployment. Ranking dominates at higher durations.

The baseline calendar time cycle ( $\alpha_{2}$ 's) and the cycle in the interaction effect $\left(\alpha_{3}\right.$ 's) are in phase in the entire data period. Thus, we find no evidence of asymmetries of the interaction effect of the business cycle. However, as is shown by Figure 2, both cycles closely track each other in the first half of the data period, whereas the interaction cycle is substantially lower in the 1980s. We interpret this remarkable shift as a movement towards less duration dependence at low durations and more duration dependence at higher durations in the early 1980 s.

\subsection{The composition of the inflow}

So far, we have not allowed for cyclical fluctuations depending on the moment of inflow, $\tau-t$, other than shifts in the shares of males and females and blacks and whites. Such fluctuations may be caused by changes in the composition of the inflow into unemployment over the business cycle. As indicated in the introduction the literature is not clear about the importance of this effect.

As argued before, interaction between duration and cycle can be due to changes in the composition of the inflow across the cycle. We will now attempt to inquire to what extent this is true. As a robustness test of our model, and with the qualifications of Appendix $\mathrm{C}$, we have re-estimated both the preferred flexible model in Column 1 of Table 1 and the model with $\psi_{2, c}(\tau)$ depending on the help wanted index, allowing in both models for variation of $\psi_{3, c}(\tau-t)$ over the business cycle. We specify $\psi_{3, c}(\tau-t)=\exp \left(\beta_{3} c(\tau-t)\right)$, where $c(\tau-t)$ is again the help wanted index. Because of the non-identification of an exponential trend in $\psi_{3, c}(\tau-t)$, we do not add a linear trend term, nor do we specify $\psi_{3, c}(\tau-t)$ as a polynomial series in $\tau-t$.

Table 2 shows the estimation results. Both specifications produce strong procyclical effects of changes in the composition of the inflow on the exit probability 
from unemployment, which is consistent with the results of Darby, Haltiwanger and Plant (1985). The average quality of the inflow in booms is higher than the average quality of the inflow in slumps. Because of this the average duration of unemployment in booms is shorter than the average duration in slumps, even if individual unemployment durations distributions do not change.

Most parameter estimates are not strongly affected by the introduction of cyclical variation in the composition of the inflow. The dummies for the demographic groups, the duration dependence estimates, the seasonal effects in the outflow and the seasonal effects due to the composition of the inflow are practically the same. The coefficients of the interaction effects are somewhat affected, but the result that there is negative interaction for lower duration classes and positive interaction for higher duration classes does not change. Only the coefficients of the calendar time effect in the outflow seem to be affected. The coefficient of the help-wanted index is $35 \%$ smaller in the second column of Table 2 than it is in the corresponding column of Table 1 . So, allowing for compositional variation in the inflow reduces the estimated instantaneous calendar time effects in the outflow. Still, a substantial cyclical variation is left in the instantaneous effect on the outflow from unemployment. Since the coefficients $\beta_{2}$ and $\beta_{3}$ have about the same size we conclude that the instantaneous effect of calendar time and the composition effect are about the same.

\section{Decomposition of unemployment dynamics}

So far, we have studied the nature of interaction between the business cycle and exit probabilities from unemployment in isolation from the incidence, and the level of unemployment itself. In this section, we investigate the role of the business cycle, duration dependence, and interaction in explaining fluctuations of the aggregate unemployment rate. Point of departure are the estimation results from Table 1, Column 1. In Subsection 5.1 we focus on the most basic decomposition of unemployment in incidence and duration. Subsection 5.2 then zooms in on the role of compositional effects.

\subsection{Incidence versus duration}

From the definition of the exit probability we can derive a decomposition of unemployment at calendar time $\tau$,

$$
U_{g}(\tau)=U_{g}(0 ; \tau)+\sum_{t=1}^{\infty} U_{g}(0 ; \tau-t) \prod_{i=0}^{t-1}\left(1-\theta_{g}(i ; \tau-t+i)\right)
$$


In steady state, when all variables are constant over calendar time $\tau$, this decomposition reduces to

$$
U_{g}=U_{g}(0)\left[1+\sum_{t=1}^{\infty} \prod_{i=0}^{t-1}\left(1-\theta_{g}(i)\right)\right],
$$

where we have dropped the argument $\tau$ for obvious reasons. We will refer to the number of newly unemployed as unemployment 'incidence' and to the term between brackets as unemployment 'duration'.

Equation (23) implies that, in a steady state, relative changes of the unemployment rate equal the sum of relative changes in unemployment incidence and relative changes in unemployment duration. In turn, changes in unemployment durations are one-to-one related to the exit probabilities from unemployment. For example, in absence of duration dependence- say that the exit probability equals some constant $\bar{\theta}_{g}$ - unemployment duration is the reciprocal of this probability. In that specific case, unemployment is given by $U_{g}(0) / \bar{\theta}_{g}$. Then, the (partial) elasticities of unemployment with respect to the incidence and the exit probability equal 1 and -1 , respectively. Obviously, equation (22) shows that, out of steady state, unemployment adjusts slowly to changes in the incidence and the exit probability. Furthermore, compared to the comparative static effects, transitory changes in the underlying components only partially translate in changes in unemployment.

Three hurdles have to be taken before we can use our estimation results of the interaction model of unemployment duration from Subsection 4.1 to decompose fluctuations in the aggregate unemployment rate.

First, we have to incorporate data on the size of the labor force for which we use CPS data on group $g$ (all groups), say $L_{g}(\tau)(L(\tau))$, and 12-month moving averages of these statistics, say $L_{g, c}(\tau)\left(L_{c}(\tau)\right)$, to transform cyclical and trend fluctuations in unemployment into unemployment rate fluctuations.

Second, we need data on incidence before we can apply (22). As we want to separate seasonal effects from longer run developments in the incidence, incidence is also modeled as the product of a seasonal and a long run term,

$$
U_{g}(0 \mid \tau)=\omega_{g, 4}(\tau) \psi_{g, 4, c}(\tau)
$$

where $\omega_{g, 4}(\tau)$ and $\psi_{g, 4, c}(\tau)$ are specified like the seasonal and cyclical terms in the interaction model. Note that we specify different models for different demographic groups to allow for shifts in the shares of these groups in the inflow into unemployment. An empirical specification can be found by recalling (7) for $t=0$, and taking $\operatorname{logs}$ : $\log \widetilde{U}_{g}(0 \mid \tau)=\log U_{g}(0 \mid \tau)+\log \varepsilon_{g, t, \tau}$. Estimated cycles 
in the incidence rate, $\log \psi_{g, 4, c}(\tau) / L_{g, c}(\tau)$, are plotted in Figure 5. A comparison with Figure 1 learns that incidence is countercyclical, with male incidence being the most sensitive to the cycle. Furthermore, there is a shift towards a higher proportion of males in the inflow into unemployment. This even holds after correction for the increased share of females in the labor force. Clearly, both developments may have an impact on aggregate unemployment, as males have longer unemployment durations.

Third, as mentioned in Section 3, we have not modeled exit probabilities for high duration classes. Consequently, we can not simulate $U_{g}(\tau)$ as it is given by equation (22). Instead, we will focus on unemployment in the first six duration classes, or more precisely, on the rate of unemployment in the first six duration classes, $u_{6}(\tau)=\sum_{g} \sum_{t=0}^{5} U_{g}(t \mid \tau) / L(\tau)$. Appendix B provides details on the decomposition procedure. So, in the remainder of this subsection where we use the term 'unemployment rate' we in fact mean the unemployment rate based on the first 6 months of unemployment, which includes approximately $90-95 \%$ of all unemployment spells.

We graphically illustrate the importance of the various components of unemployment dynamics. To start with, Figure 6 shows that the evolution of the actual unemployment rate is well represented by the cyclical part of the estimated model.

Figure 7 shows the effect of omitting the interaction cycle from the estimated model. It appears that the amplitudes of the cycle are somewhat larger without the interaction cycle. Figure 7 also shows that the amplitude of the unemployment rate cycle is reduced by approximately one half if we omit both the interaction and the baseline cycles, and thus the instantaneous cyclical effect on the outflow. Figure 8 shows the effect of the incidence on the cyclical pattern of the unemployment rate. If the cyclical variation in the incidence is removed the amplitude of the cycle again reduces substantially. The contributions of incidence and duration to the variation of the unemployment rate over the business cycle are about the same.

Figure 9 compares the full cycle fluctuations in the unemployment rate with unemployment rate fluctuations generated in the absence of duration dependence. As is explained in Appendix B, the average, or 'steady state', exit probability is fixed at a value that generates the same (truncated) expected duration as the original set of exit probabilities averaged over calendar time. The interaction effects are switched off. Almost all of the, fairly small, changes in the cyclical pattern of the unemployment rate appear to be due to fixing the interaction effect. Clearly, individual duration dependence per se is only of second order importance 
compared to the driving changes in incidence and overall outflow probabilities.

\subsection{Compositional variation and aggregate unemployment}

We capture part of the cyclical variation in the composition of the inflow by allowing for a changing demographic composition of unemployment incidence. We capture the remaining variation, which by itself is unobserved, by introducing a relationship between the composition of the inflow and the help-wanted index (see Subsection 4.2). Figure 10 shows the effect of fixing the demographic composition of the inflow. As could be expected from the observed time pattern of the male-female incidence shares and the difference between male and female exit probabilities, variation in the composition of the inflow explains some of both the cyclical fluctuations in and the secular rise of unemployment. This is in conflict with some of the earlier literature discussed in the introduction. The effect of the remaining cyclical variation in the composition of the inflow, for which we used the help-wanted index as an indicator, is more difficult to illustrate. Basically the help-wanted index is only indirectly related to the composition of the inflow. Whereas there is a direct relation between the help-wanted index and the outflow from unemployment because of the matching process between job vacancies and unemployed, in case of the composition of the inflow the help-wanted index is just an indicator. There is no reason why the composition of the inflow would be in any way related to the number of job vacancies. Therefore, we can only tentatively indicate the effect of the unobserved cyclical variation in the composition of the inflow. As shown in Tables 1 and 2 the introduction of a compositional effect connected to the help-wanted index reduces the direct effect of the business cycle on the unemployment exit rate. Both effects have about the same magnitude which implies that the two competing explanations of fluctuations in average unemployment duration are in fact equally valid.

\section{Conclusions}

In this paper we present a full and integrated decomposition of U.S. unemployment dynamics. In some respects we get conventional results, in other respects we get brand-new results. And, our results are achieved within a very general modelling framework.

We find that unemployment durations at the individual level are shorter in booms and longer in slumps. Furthermore, unemployment incidence is countercyclical, while the changing composition of the inflow has procyclical effects. 
Thus, cyclical variations in U.S. unemployment are driven by the combined effects of variations in individual exit probabilities, incidence and a changing composition of the inflow into unemployment. Variations in unemployment duration seem to explain about half of all variations, while the incidence explains the other half. The cyclical variations in unemployment duration themselves are caused by both an instantaneous effect and by the effect of a changing composition of the inflow into unemployment. Roughly speaking, both account for about half of the duration effect. It should however be noted that the compositional effect, to the extent in which it concerns unobserved characteristics, is identified from functional form restrictions.

There are also substantial seasonal fluctuations in U.S. unemployment. Exit probabilities are low in the first and high in the third quarter. Furthermore, unemployed entering in the first quarter have relatively short unemployment spells, whereas unemployed entering in the third quarter experience relatively long spells. As unemployment incidence is relatively small in the first and large in the third quarter, we conclude that this may be related to competition among unemployed.

Finally, we find that the individual exit probabilities out of unemployment decline over the duration of the unemployment spell. This negative duration dependence is a quantitatively unimportant determinant of unemployment variation.

We establish that sorting is the dominant explanation of the observed interaction between the business cycle and duration in the first two months of unemployment. Consequently, at low durations the cyclical sensitivity of exit probabilities increases with duration. At higher durations, however, the sign of this interaction effect changes, and cyclical sensitivity increases fast with duration. It turns out that this is only consistent with the presence of ranking. It should be noted that the interaction effect, to the extent in which it cannot be attributed to a changing composition, is a quantitatively unimportant determinant of unemployment variation across the cycle.

From the perspective of our model of sorting and ranking we conclude that there is considerable heterogeneity in unemployment duration distributions between labor market segments. Clearly, this leaves some scope for identifying segments and directing labor market policies to those segments that perform badly. On top of this, our analysis shows that, even at a high level of aggregation, within market ranking eventually dominates sorting effects, and is thus of considerable importance as well. The institutional structure of the current U.S. unemployment benefit system seems to account for the consequences of the ranking phenomenon because the length of the unemployment benefit entitlement period depends on 
the business cycle. Labor market policies directed at long term unemployment in general can be beneficial. Furthermore, even if ranking is not induced by substantial loss of skills, it may demotivate long term unemployed, and thus call for similar policies. As ranking is associated with limited adjustment of wages to unemployment, such policies could also be effective in reducing persistence of unemployment at the aggregate level. 


\section{References}

Abbring, J.H., G.J. van den Berg, and J.C. van Ours (1994), "The anatomy of unemployment dynamics", Working paper, Tinbergen Institute, Amsterdam/Rotterdam.

Abbring, J.H., G.J. van den Berg, and J.C. van Ours (1997), "Business cycles and compositional variation in U.S. Unemployment", Working paper, Tinbergen Institute, Amsterdam/Rotterdam.

Abowd, J.M. and A. Zellner (1985), "Estimating gross labor-force flows", Journal of Business and Economic Statistics, 3, 254-283.

Abramowitz, M. and I.A. Stegun, editors (1970), Handbook of Mathematical Functions; With Formulas, Graphs and Mathematical Tables, Dover Publications, New York.

Akerlof, G.A. and B.G.M. Main (1980), "Unemployment spells and unemployment experience", American Economic Review, 70, 885-893.

Akerlof, G.A. and B.G.M. Main (1981), "An experience-weighted measure of employment and unemployment durations", American Economic Review, 71, 1003-1011.

Akhiezer, N.I. (1965), The Classical Moment Problem, Oliver and Boyd, Edinburgh.

Baker, M. (1992a), "Unemployment duration: Compositional effects and cyclical variability", American Economic Review, 82 , 313-321.

Baker, M. (1992b), "Digit preferences in CPS unemployment data", Economics Letters, 39, 117-121.

Blanchard, O.J. (1991), "Wage bargaining and unemployment persistence", Journal of Money, Credit, and Banking, 23, 277-292.

Blanchard, O.J. (1996), "The plight of the long term unemployed", Working paper, MIT.

Blanchard, O.J. and P.A. Diamond (1994), "Ranking, unemployment duration, and wages", Review of Economic Studies, 61 , 417-434.

Butler, R.J. and J.B. McDonald (1986), "Trends in unemployment duration data", Review of Economics and Statistics, 68, 545-557. 
Carlson, J.A. and M.W. Horrigan (1983), "Measures of unemployment duration as guides to research and policy: comment", American Economic Review, $73,1143-1150$.

Clark, K.B. and L.H. Summers (1979), "Labor market dynamics and unemployment: a reconsideration", Brookings Papers on Economic Activity , 12-60.

Darby, M.R., J. Haltiwanger, and M. Plant (1985), "Unemployment rate dynamics and persistent unemployment under rational expectations", American Economic Review, 75, 614-637.

Devine, T.J. and N.M. Kiefer (1991), Empirical Labor Economics; the Search Approach, Oxford, Oxford University Press.

Dynarski, M. and S.M. Sheffrin (1990), "The behavior of unemployment durations over the cycle", Review of Economics and Statistics, 72, 350-356.

Imbens, G.W. and L.M. Lynch (1992), "Labor market transitions over the business cycle", Working paper, Harvard University.

Kaitz, H. (1970), "Analyzing the length of spells of unemployment", Monthly Labor Review, 93, 11-20.

Lebon, I. (1993), "Hiring policies of firms and long term unemployment", Working paper, Université de Paris I.

Shohat, J.A. and J.D. Tamarkin (1943), The Problem of Moments, American Mathematical Society, New York.

Sider, H. (1985), "Unemployment duration and incidence: 1968-82", American Economic Review, 75, 461-472.

Van den Berg, G.J. and J.C. van Ours (1994), "Unemployment dynamics and duration dependence in France, The Netherlands, and The United Kingdom", Economic Journal, 104, 432-443.

Van den Berg, G.J. and J.C. van Ours (1996), "Unemployment dynamics and duration dependence", Journal of Labor Economics, 14, 100-125.

Van den Berg, G.J. and J.C. van Ours (1997), "Eyeballing state dependence and unobserved heterogeneity in aggregate unemployment duration data", Research in Labor Economics, forthcoming. 


\section{Appendices}

\section{A The sorting process}

This appendix provides details on the sorting model in discrete time. We use discrete duration $t$ and discrete calendar time $\tau$, measured on the time scales introduced in Subsection 2.3. Let $c_{\tau}$ denote the value of the business cycle indicator at calendar time $\tau$, and let $\vartheta\left(t \mid c_{\tau}, v\right)$ now denote the exit probability from unemployment at calendar time $\tau$ and duration $t$. Assume that $G(v)$ is absolutely continuous, and denote the density of $v$ by $g(v)$. We again assume that $\operatorname{Pr}\left[0<\vartheta\left(t \mid c_{\tau}, v\right)<1\right]=1$ and $\partial \eta\left(c_{\tau}, t\right) / \partial c_{\tau}>0$. Denote $\nu(\tau, t)=$ $\mathbb{E}\left[v \mid\{c\}_{\tau-1}, \mathbf{t} \geq t\right]$, where $\{c\}_{\tau-1}=\left\{\ldots, c_{\tau-2}, c_{\tau-1}\right\}$. The conditional survivor function is given by $S(t \mid \tau, v):=\operatorname{Pr}\left(\mathbf{t} \geq t \mid\{c\}_{\tau-1}, v\right)=\prod_{i=0}^{t-1}\left(1-\eta\left(c_{\tau-t+i}, i\right) v\right)$, where we adopt the convention $\prod_{i=0}^{-1} x_{i}=1$. The unconditional survivor function equals $S(t \mid \tau)=\mathbb{E}[S(t \mid \tau, v)]=\mathbb{E}\left[\prod_{i=0}^{t-1}\left(1-\eta\left(c_{\tau-t+i}, i\right) v\right)\right]$, where expectations are taken with respect to $G(v)$, unless conditioning is explicitly denoted. The distribution of $v$ conditional on survival up to $t$ is given by

$$
\begin{aligned}
\operatorname{Pr}\left(\mathbf{v} \leq v \mid\{c\}_{\tau-1}, \mathbf{t} \geq t\right) & =\frac{\operatorname{Pr}\left(\mathbf{v} \leq v, \mathbf{t} \geq t \mid\{c\}_{\tau-1}\right)}{S(t \mid \tau)} \\
& =\frac{\int_{0}^{v} S(t \mid \tau, \mathbf{v}) \mathrm{d} G(\mathbf{v})}{S(t \mid \tau)} .
\end{aligned}
$$

Therefore, the conditional density of $v$, say $g(v \mid \tau, \mathbf{t} \geq t)$, is given by

$$
\begin{aligned}
g(v \mid \tau, \mathbf{t} \geq t) & =\frac{S(t \mid \tau, v)}{S(t \mid \tau)} g(v) \\
& =\frac{\prod_{i=0}^{t-1}\left(1-\eta\left(c_{\tau-t+i}, i\right) v\right)}{\mathbb{E}\left[\prod_{i=0}^{t-1}\left(1-\eta\left(c_{\tau-t+i}, i\right) v\right)\right]} g(v) .
\end{aligned}
$$

Thus, the $k$-th moment of $v$, conditional on survival up to time $t$, is given by

$$
\mathbb{E}\left[v^{k} \mid\{c\}_{\tau-1}, \mathbf{t} \geq t\right]=\frac{\mathbb{E}\left[v^{k} \prod_{i=0}^{t-1}\left(1-\eta\left(c_{\tau-t+i}, i\right) v\right)\right]}{\mathbb{E}\left[\prod_{i=0}^{t-1}\left(1-\eta\left(c_{\tau-t+i}, i\right) v\right)\right]}
$$

Now, impose steady state on $\eta\left(c_{\tau}, t\right)$, such that $\eta\left(c_{\tau}, t\right)=\eta(c)$ for each $\tau$ and $t$, and some constant $c=c_{\tau}$, and $\nu(\tau, t)=\nu(c, t)$. Then, we can use equation (27) to show that

$$
\nu(c, t)=\frac{\mathbb{E}\left[v(1-\eta(c) v)^{t}\right]}{\mathbb{E}\left[(1-\eta(c) v)^{t}\right]} .
$$


Then, duration dependence between duration classes $t$ and $t+1$ can be characterized by $\nu(c, t+1)-\nu(c, t)$. It can be shown that

$$
\nu(c, t+1)-\nu(c, t)=-\eta(c) \frac{S(t \mid c)}{S(t+1 \mid c)} \operatorname{var}(v \mid c, \mathbf{t} \geq t)
$$

Thus, if the (conditional) variance of $v$ is strictly positive, the expected value of $v$ falls between cohorts in duration classes $t$ and $t+1$. This is the familiar result that unobserved heterogeneity generates negative duration dependence in observed exit probabilities. The marginal effect of a change in $c$ on duration dependence between 0 and $t$ is given by

$$
\begin{aligned}
& \frac{\partial}{\partial c} \log \frac{\nu(c, t)}{\nu(c, 0)} \\
= & -\eta_{c}(c) t\left\{\frac{\mathbb{E}\left[v^{2}(1-\eta(c) v)^{t-1}\right]}{\mathbb{E}\left[v(1-\eta(c) v)^{t}\right]}-\frac{\mathbb{E}\left[v(1-\eta(c) v)^{t-1}\right]}{\mathbb{E}\left[(1-\eta(c) v)^{t}\right]}\right\} \\
= & -\frac{\eta_{c}(c) t}{1-\eta(c) \mathbb{E}[v \mid c, \mathbf{t} \geq t-1]} \times \\
& \left\{\frac{1-\eta(c) \mathbb{E}[v \mid c, \mathbf{t} \geq t-1]}{1-\eta(c) \mathbb{E}\left[v^{2} \mid c, \mathbf{t} \geq t-1\right] / \mathbb{E}[v \mid c, \mathbf{t} \geq t-1]} \frac{\mathbb{E}\left[v^{2} \mid c, \mathbf{t} \geq t-1\right]}{\mathbb{E}[v \mid c, \mathbf{t} \geq t-1]}-\mathbb{E}[v \mid c, \mathbf{t} \geq t-1]\right\},
\end{aligned}
$$

which is weakly smaller than 0 as $\mathbb{E}\left[v^{2} \mid c, \mathbf{t} \geq t-1\right] \geq \mathbb{E}[v \mid c, \mathbf{t} \geq t-1]^{2}$ and $\eta(c) \mathbb{E}\left[v^{2} \mid c, \mathbf{t} \geq t-1\right]<\mathbb{E}[v \mid c, \mathbf{t} \geq t-1]$ (Shohat and Tamarkin, 1943, and Akhiezer, 1965). If the first inequality holds strictly, i.e. if $\operatorname{var}(v \mid c, \geq t-1)>0$, the interaction effect is strictly negative, which proves the claim in the main text.

Finally, note that qualitatively similar results can be derived in a continuous time sorting model. Abbring, Van den Berg and Van Ours (1997) provide details.

\section{B Details on the decomposition}

The decomposition in Section 5 is computed as follows. Note that we can logtransform (9) and (24) into

$$
\begin{aligned}
\bar{\theta}_{g}(t \mid \tau)= & \alpha_{0, g}+\bar{\psi}_{1}(t)+\bar{\psi}_{2, c}(\tau)+\bar{\omega}_{2}(\tau) \\
& +\bar{\psi}_{3, c}(\tau-t)+\bar{\omega}_{3}(\tau-t)+\bar{\psi}_{3, i}(t, \tau) \text { and } \\
\bar{U}_{g}(0 \mid \tau)= & \bar{\omega}_{g, 4}(\tau)+\bar{\psi}_{g, 4, c}(\tau),
\end{aligned}
$$


where bars denote logs. In the decompositional exercises, one or more terms in (31) are fixed over calendar time, or, in case of the incidence, fixed between groups. Furthermore, in some cases, duration dependence is fixed at a steady state level. Suppose, we want to simulate $U(t \mid \tau)$ for $t=0, \ldots, n_{t}$ and $\tau=$ $\tau_{0} \ldots \tau_{0}+n_{\tau}-1$. We can distinguish the following cases:

1. Let $\bar{\psi}_{3, i}(\cdot, \tau):=n_{t}^{-1} \sum_{t} \bar{\psi}_{3, i}(t, \tau)$ and $\bar{\psi}_{3, i}(t, \cdot):=n_{\tau}^{-1} \sum_{\tau} \bar{\psi}_{3, i}(t, \tau)$. If interaction effects are excluded, $\psi_{3, i}(t, \tau) /\left[\psi_{3, i}(\cdot, \tau) \psi_{3, i}(t, \cdot)\right]$ is fixed at its overall geometric duration and calendar time mean. Alternatively, we could say that $\bar{\psi}_{3, i}(t, \tau)-\bar{\psi}_{3, i}(\cdot, \tau)-\bar{\psi}_{3, i}(t, \cdot)$ is fixed at its mean.

2. If seasonal effects are excluded, $\bar{\omega}_{2}(\tau), \bar{\omega}_{3}(\tau-t)$ and $\bar{\omega}_{4}(\tau)$ are fixed at their respective means. Similarly, if cyclical effects on the composition or the size of the inflow are excluded, $\bar{\psi}_{3, c}(\tau-t)$ and $\bar{\psi}_{4, c}(\tau)$ are fixed at their mean values, respectively. In case $\psi_{4, c}(\tau)$ is fixed, $L_{c}(\tau)$ is also fixed at its geometric mean.

3. If cyclical effects on the outflow are excluded $\bar{\psi}_{2, c}(\tau)+\bar{\psi}_{3, i}(\cdot, \tau)$ is fixed at its mean value, and interaction effects are fixed as explained before.

4. If we fix all functions but duration dependence, $\psi_{1}(t) \psi_{3, i}(t, \cdot)$, at their geometric means, we can compute a series of 'steady state' exit probabilities $\theta_{g}(t)$, for $t=0, \ldots, n_{t}-1$. If we exclude duration dependence from our simulations, we again fix interaction effects, and fix the 'steady state' exit probability series at the single level that corresponds to the same (truncated) expected duration as the 'steady state' series $\theta_{g}(t), t=0, \ldots, n_{t}-1$. Thus, for each group $g$, we try to find a single real root $0<\theta_{g}<1$ of

$$
1+\sum_{t=1}^{n_{t}} \prod_{i=0}^{t-1}\left[1-\theta_{g}(i)\right]=\theta_{g}^{-1}\left[1-\left(1-\theta_{g}\right)^{n_{t}+1}\right] .
$$

Then, we fix $\bar{\psi}_{1}(t)+\bar{\psi}_{3, i}(t, \cdot)$ at $\bar{\theta}_{g}$ minus the group specific geometric mean of $\bar{\theta}_{g}(t \mid \tau)-\bar{\psi}_{1}(t)-\bar{\psi}_{3, i}(t, \cdot)$.

5. Finally, we fix the shares of observed groups in the inflow by first computing average shares, say $s_{g}$, of each group $g$ in the geometric mean of the incidence. Then, we compute a representative incidence cycle from $\prod_{g} \psi_{g, 4, c}^{s_{g}}$. 


\section{Identification and the interpretation of the interaction effect}

Recall from Subsection 2.3 that our model is given by

$$
\theta(t \mid \tau)=\exp \left(\alpha_{0}\right) \psi_{1}(t) \psi_{2, c}(\tau) \psi_{3, c}(\tau-t) \psi_{3, i}(t, \tau)
$$

where we drop the subscript $g$ and seasonal effects for the moment. Our model is fully identified if we can uniquely determine $\alpha_{0}, \psi_{1}(t), \psi_{2, c}(\tau), \psi_{3, c}(\tau-t)$, and $\psi_{3, i}(t, \tau)$, for all $t \geq 0$ and $\tau \geq \tau_{0}$. In this appendix we show under which conditions our model is identified.

As argued before, our decomposition is very flexible, allowing not only for duration and calendar time dependence, but also for interaction effects between duration dependence and calendar time, and dependence on the moment of inflow. To identify the model only one additional assumption is required. ${ }^{12}$

We have to rule out specifications of $\psi_{3, c}(\tau-t)$ from which a multiplicative exponential trend can be separated. Abbring, Van den Berg and Van Ours (1994) show that without this restriction, $\psi_{1}(t)$ and $\psi_{2, c}(\tau)$ on the one hand, and $\psi_{3, c}(\tau-t)$ on the other, cannot be separated. ${ }^{13}$ Therefore, the minimum assumption that we have to make is that $\psi_{3, c}(\tau-t)$ does not contain an exponential trend. This assumption seems fairly innocuous. In fact, in our baseline analysis we assume that there is no long run variation in the composition of the inflow into unemployment. Subsequently, in an additional analysis we investigate an alternative specification, in which $\psi_{3, c}(\tau-t)$ is allowed to vary over the cycle.

\footnotetext{
${ }^{12}$ Note that we can arbitrarily normalize 4 of the 5 components of the r.h.s. of (33), say by putting $\psi_{1}(\bar{t})=\psi_{2}(\bar{\tau})=\psi_{3, c}(\bar{\tau}-\bar{t})=\psi_{3, i}(\bar{t}, \bar{\tau})=1$. Then, $\alpha_{0}$ accounts for the scale of $\theta(t \mid \tau)$, and $\theta(\bar{t} \mid \bar{\tau})$ equals $\exp \left(\alpha_{0}\right)$. Also note that in Subsection 2.3 we restricted the functional form of $\psi_{3, i}(t, \tau)$ to satisfy $\psi_{3, i}(\bar{t}, \tau)=\psi_{3, i}(t, \bar{\tau})=1$. Without this assumption, $\psi_{3, i}(t, \tau)$ is allowed to contain a multiplicative component that only depends on the moment of inflow $\tau-t$. Clearly, such a factor cannot be distinguished from $\psi_{3, c}(\tau-t)$. It can easily be seen that the indeterminacy is solved by this assumption. Suppose that we have a model $\left\{\psi_{1}(t), \psi_{2}(\tau), \psi_{3, c}(\tau-t), \psi_{3, i}(t, \tau)\right\}$. Now, let $\tilde{\psi}_{3, c}(\tau-t) \equiv 1$ and $\tilde{\psi}_{3, i}(t, \tau)=\psi_{3, i}(t, \tau) \psi_{3, c}(\tau-t)$. Clearly, $\tilde{\psi}_{3, i}(t, \tau)$ is indeed a function of $t$ and $\tau$ for which $\tilde{\psi}_{3, i}(\bar{t}, \bar{\tau})=1$. It can easily be seen that our original model and $\left\{\psi_{1}(t), \psi_{2}(\tau), \tilde{\psi}_{3, c}(\tau-t), \tilde{\psi}_{3, i}(t, \tau)\right\}$ are observationally equivalent. However, the specification of $\tilde{\psi}_{3, i}(t, \tau)$ violates the new assumption, unless $\psi_{3, c}(\tau-t)=1$ for all $\tau-t$.

${ }^{13}$ Such specifications can be characterized by $h(\tau-t) \exp [\gamma(\tau-t)]$, with $h(\tau-t)$ nonmultiplicative in $\tau$ and $t$. Clearly, if $\psi_{3, c}(\tau-t)$ could be separated this way, a model $\left\{\tilde{\psi}_{1}(t), \tilde{\psi}_{2}(\tau), \tilde{\psi}_{3, c}(\tau-t), \psi_{3, i}(t, \tau)\right\}$ with duration dependence $\tilde{\psi}_{1}(t)=\psi_{1}(t) \exp (-\gamma t)$, calendar time dependence $\tilde{\psi}_{2, c}(\tau)=\psi_{2, c}(\tau) \exp (\gamma \tau)$, and moment of inflow dependence $\tilde{\psi}_{3, c}(\tau-t)=$ $h(\tau-t)$ would be observational equivalent to a model $\left\{\psi_{1}(t), \psi_{2}(\tau), \psi_{3, c}(\tau-t), \psi_{3, i}(t, \tau)\right\}$.
} 
We end this subsection by discussing the interpretation of the interaction effect $\psi_{3, i}(t, \tau)$ as representation of the dynamic effects of ranking and sorting. From the sorting model of Subsection 2.2 we know that interaction effects stemming from sorting concern interaction between the level of the exit probability, represented by the cycle $c$, and duration dependence. In a model in which the exit probabilities do not depend on the moment of inflow in the longer run, and thus $\psi_{3, c}(\tau-t) \equiv 1$, this can easily be translated in terms of the empirical model: the role of the cycle $c$ is taken by $\psi_{2, c}(\tau)$, so this function should somehow enter in the interaction effect. Then, if $\psi_{2, c}(\tau)$ is high, exit probabilities are high, and any existing sorting effects are stronger. The converse holds for ranking.

However, if the longer run level of the exit probabilities depends on both the outflow cycle $\psi_{2, c}(\tau)$ and the inflow cycle $\psi_{3, c}(\tau-t)$ it is the cumulative effect of both these cycles that matters. Both in the top of the inflow and in the top of outflow cycle, the exit probabilities will be relatively large, and any sorting effects will be strong. As the overall level of the exit probabilities matters for the interaction effect, both cycles should appear in the interaction term.

Thus, in designing a parameterization of the model of Subsection 2.3, we either have to rule out cyclical fluctuations in the composition of the inflow, or allow for sufficient flexibility in the dependence of $\psi_{3, i}(t, \tau)$ on calender time $\tau$. Sufficient flexibility is achieved if the interaction effect is able to capture interaction between the overall level of the exit probabilities and duration dependence. 
Table 1: Interaction model; estimation results

\begin{tabular}{|c|c|c|c|c|c|c|c|c|}
\hline \multicolumn{9}{|c|}{ observed individual characteristics } \\
\hline$\alpha_{0 b m}$ & -0.75 & $(0.01)$ & -0.77 & $(0.02)$ & -0.75 & $(0.01)$ & -0.69 & $(0.01)$ \\
\hline$\alpha_{0 b f}$ & -0.63 & $(0.01)$ & -0.65 & $(0.02)$ & -0.63 & $(0.01)$ & -0.57 & $(0.01)$ \\
\hline$\alpha_{0 w m}$ & -0.71 & $(0.01)$ & -0.73 & $(0.02)$ & -0.71 & $(0.01)$ & -0.65 & $(0.01)$ \\
\hline$\alpha_{0 w f}$ & -0.59 & $(0.01)$ & -0.61 & $(0.02)$ & -0.59 & $(0.01)$ & -0.53 & $(0.01)$ \\
\hline \multicolumn{9}{|c|}{ baseline duration dependence } \\
\hline$\psi_{11}$ & -0.21 & $(0.02)$ & -0.17 & $(0.02)$ & -0.19 & $(0.01)$ & -0.21 & $(0.01)$ \\
\hline$\psi_{12}$ & -0.39 & $(0.02)$ & -0.35 & $(0.03)$ & -0.38 & $(0.01)$ & -0.38 & $(0.02)$ \\
\hline$\psi_{13}$ & -0.62 & $(0.02)$ & -0.59 & $(0.03)$ & -0.64 & $(0.02)$ & -0.58 & $(0.02)$ \\
\hline$\psi_{14}$ & -1.12 & $(0.06)$ & -1.15 & $(0.05)$ & -1.23 & $(0.04)$ & -1.06 & $(0.04)$ \\
\hline \multicolumn{9}{|c|}{ baseline cycle outflow } \\
\hline$\beta_{2}$ & \multicolumn{6}{|c|}{ (help wanted index) } & 0.76 & $\overline{(0.06)}$ \\
\hline$\alpha_{21}$ & -0.12 & $(0.01)$ & -0.09 & $(0.04)$ & -0.08 & $(0.01)$ & -0.12 & $(0.01)$ \\
\hline$\alpha_{22}$ & 0.04 & $(0.01)$ & 0.03 & $(0.03)$ & 0.04 & $(0.01)$ & & \\
\hline$\alpha_{23}$ & -0.06 & $(0.01)$ & -0.05 & $(0.03)$ & -0.04 & $(0.01)$ & & \\
\hline$\alpha_{24}$ & -0.05 & $(0.01)$ & -0.03 & $(0.03)$ & -0.03 & $(0.01)$ & & \\
\hline$\alpha_{25}$ & -0.04 & $(0.01)$ & -0.03 & $(0.03)$ & -0.02 & $(0.01)$ & & \\
\hline$\alpha_{26}$ & -0.02 & $(0.01)$ & -0.01 & $(0.02)$ & -0.01 & $(0.01)$ & & \\
\hline$\alpha_{27}$ & 0.03 & $(0.01)$ & 0.01 & $(0.03)$ & 0.03 & $(0.01)$ & & \\
\hline$\alpha_{28}$ & 0.02 & $(0.01)$ & 0.00 & $(0.02)$ & 0.02 & $(0.01)$ & & \\
\hline$\alpha_{29}$ & 0.02 & $(0.01)$ & 0.01 & $(0.02)$ & 0.01 & $(0.00)$ & & \\
\hline$\alpha_{210}$ & -0.02 & $(0.01)$ & -0.02 & $(0.02)$ & -0.02 & $(0.00)$ & & \\
\hline$\alpha_{211}$ & 0.04 & $(0.01)$ & 0.01 & $(0.01)$ & 0.03 & $(0.00)$ & & \\
\hline$\alpha_{212}$ & 0.04 & $(0.01)$ & 0.01 & $(0.01)$ & 0.03 & $(0.00)$ & & \\
\hline$\alpha_{213}$ & -0.04 & $(0.01)$ & -0.03 & $(0.01)$ & -0.03 & $(0.00)$ & & \\
\hline$\alpha_{214}$ & -0.00 & $(0.00)$ & -0.00 & $(0.01)$ & 0.00 & $(0.00)$ & & \\
\hline$\alpha_{215}$ & 0.03 & $(0.01)$ & 0.01 & $(0.01)$ & 0.02 & $(0.00)$ & & \\
\hline \multicolumn{9}{|c|}{ seasonal effect outflow } \\
\hline$\omega_{22}$ & 0.07 & $(0.01)$ & 0.08 & $(0.01)$ & 0.07 & $(0.01)$ & 0.07 & $(0.01)$ \\
\hline$\omega_{23}$ & 0.21 & $(0.01)$ & 0.21 & $(0.01)$ & 0.21 & $(0.01)$ & 0.21 & $(0.01)$ \\
\hline$\omega_{24}$ & 0.04 & $(0.01)$ & 0.04 & $(0.01)$ & 0.04 & $(0.01)$ & 0.03 & $(0.01)$ \\
\hline
\end{tabular}


Table 1: (Continued)

\begin{tabular}{|c|c|c|c|c|c|c|c|c|}
\hline \multicolumn{9}{|c|}{ seasonal effect composition inflow } \\
\hline$\omega_{32}$ & -0.05 & $(0.01)$ & -0.05 & $(0.01)$ & -0.05 & $(0.01)$ & -0.05 & $(0.01)$ \\
\hline$\omega_{33}$ & -0.11 & $(0.01)$ & -0.11 & $(0.01)$ & -0.11 & $(0.01)$ & -0.11 & $(0.01)$ \\
\hline$\omega_{34}$ & -0.09 & $(0.01)$ & -0.09 & $(0.01)$ & -0.09 & $(0.01)$ & -0.09 & $(0.01)$ \\
\hline \multicolumn{9}{|c|}{ interaction; duration parameters } \\
\hline$\overline{\xi_{10}}$ & -0.37 & $(0.09)$ & & & -0.13 & $(0.09)$ & -0.30 & $(0.10)$ \\
\hline$\xi_{11}$ & 0.46 & $(0.12)$ & & & 0.41 & $(0.08)$ & 0.65 & $(0.09)$ \\
\hline$\left(\xi_{11}\right)$ & -0.37 & $(0.09)$ & & & -0.13 & $(0.09)$ & -0.30 & $(0.10)$ \\
\hline$\left(\xi_{12}\right)$ & -0.27 & $(0.08)$ & & & 0.16 & $(0.13)$ & 0.05 & $(0.15)$ \\
\hline$\left(\xi_{13}\right)$ & 0.29 & $(0.11)$ & & & 0.87 & $(0.15)$ & 1.04 & $(0.21)$ \\
\hline$\left(\xi_{14}\right)$ & 1.32 & $(0.36)$ & & & 1.99 & $(0.26)$ & 2.69 & $(0.35)$ \\
\hline \multicolumn{9}{|c|}{ interaction; cycle parameters } \\
\hline$\alpha_{31}$ & -0.17 & $(0.06)$ & -0.01 & $\overline{(0.04)}$ & & & & \\
\hline$\alpha_{32}$ & 0.01 & $(0.08)$ & 0.02 & $(0.03)$ & & & & \\
\hline$\alpha_{33}$ & 0.06 & $(0.04)$ & -0.01 & $(0.04)$ & & & & \\
\hline$\alpha_{34}$ & -0.05 & $(0.07)$ & -0.02 & $(0.02)$ & & & & \\
\hline$\alpha_{35}$ & 0.01 & $(0.03)$ & -0.00 & $(0.03)$ & & & & \\
\hline$\alpha_{36}$ & -0.03 & $(0.06)$ & -0.01 & $(0.02)$ & & & & \\
\hline$\alpha_{37}$ & 0.06 & $(0.04)$ & 0.03 & $(0.03)$ & & & & \\
\hline$\alpha_{38}$ & 0.07 & $(0.04)$ & 0.02 & $(0.02)$ & & & & \\
\hline$\alpha_{39}$ & 0.06 & $(0.05)$ & 0.00 & $(0.02)$ & & & & \\
\hline$\alpha_{310}$ & 0.03 & $(0.03)$ & -0.01 & $(0.01)$ & & & & \\
\hline$\alpha_{311}$ & 0.07 & $(0.04)$ & 0.04 & $(0.02)$ & & & & \\
\hline$\alpha_{312}$ & 0.08 & $(0.03)$ & 0.04 & $(0.01)$ & & & & \\
\hline$\alpha_{313}$ & 0.00 & $(0.03)$ & -0.02 & $(0.01)$ & & & & \\
\hline$\alpha_{314}$ & -0.02 & $(0.02)$ & 0.00 & $(0.01)$ & & & & \\
\hline$\alpha_{315}$ & 0.11 & $(0.04)$ & 0.01 & $(0.01)$ & & & & \\
\hline \multicolumn{9}{|c|}{ measurement errors } \\
\hline$\overline{\sigma_{b m}}$ & 0.23 & $(0.00)$ & 0.23 & $(0.00)$ & 0.23 & $\overline{(0.00)}$ & 0.24 & $(0.00)$ \\
\hline$\sigma_{b f}$ & 0.23 & $(0.00)$ & 0.23 & $(0.00)$ & 0.23 & $(0.00)$ & 0.24 & $(0.00)$ \\
\hline$\sigma_{w m}$ & 0.14 & $(0.00)$ & 0.14 & $(0.00)$ & 0.14 & $(0.00)$ & 0.15 & $(0.00)$ \\
\hline$\sigma_{w f}$ & 0.13 & $(0.00)$ & 0.13 & $(0.00)$ & 0.13 & $(0.00)$ & 0.14 & $(0.00)$ \\
\hline $\bar{N}$ & $288 \times$ & $4 \times 5$ & $288 \times$ & $4 \times 5$ & $288>$ & $4 \times 5$ & $288 \times$ & $4 \times 5$ \\
\hline $\log \mathcal{L}$ & 813 & 5.11 & & 9.00 & & & 781 & .42 \\
\hline
\end{tabular}

Note: standard errors in parentheses. 
Table 2: Interaction model with cyclical dependence inflow: estimation results

\begin{tabular}{|c|c|c|c|c|}
\hline \multicolumn{5}{|c|}{ observed individual characteristics } \\
\hline$\alpha_{0 b m}$ & -0.75 & $(0.01)$ & -0.68 & $(0.01)$ \\
\hline$\alpha_{0 b f}$ & -0.63 & $(0.01)$ & -0.55 & $(0.01)$ \\
\hline$\alpha_{0 w m}$ & -0.71 & $(0.01)$ & -0.64 & $(0.01)$ \\
\hline$\alpha_{0 w f}$ & -0.59 & $(0.01)$ & -0.52 & $(0.01)$ \\
\hline \multicolumn{5}{|c|}{ baseline duration dependence } \\
\hline$\psi_{11}$ & -0.21 & $(0.01)$ & -0.21 & $(0.02)$ \\
\hline$\psi_{12}$ & -0.39 & $(0.01)$ & -0.39 & $(0.01)$ \\
\hline$\psi_{13}$ & -0.62 & $(0.01)$ & -0.62 & $(0.01)$ \\
\hline$\psi_{14}$ & -1.11 & $(0.01)$ & -1.16 & $(0.01)$ \\
\hline \multicolumn{5}{|c|}{ baseline cycle outflow } \\
\hline \multirow[t]{2}{*}{$\overline{\beta_{2}}$} & \multicolumn{4}{|c|}{ (help wanted index) } \\
\hline & & & 0.49 & $(0.07)$ \\
\hline$\alpha_{21}$ & -0.15 & $(0.01)$ & -0.19 & $(0.02)$ \\
\hline$\alpha_{22}$ & 0.05 & $(0.01)$ & & \\
\hline$\alpha_{23}$ & -0.02 & $(0.01)$ & & \\
\hline$\alpha_{24}$ & -0.03 & $(0.01)$ & & \\
\hline$\alpha_{25}$ & -0.02 & $(0.01)$ & & \\
\hline$\alpha_{26}$ & -0.01 & $(0.01)$ & & \\
\hline$\alpha_{27}$ & 0.00 & $(0.01)$ & & \\
\hline$\alpha_{28}$ & 0.00 & $(0.01)$ & & \\
\hline$\alpha_{29}$ & 0.01 & $(0.01)$ & & \\
\hline$\alpha_{210}$ & -0.01 & $(0.01)$ & & \\
\hline$\alpha_{211}$ & 0.03 & $(0.01)$ & & \\
\hline$\alpha_{212}$ & 0.00 & $(0.01)$ & & \\
\hline$\alpha_{213}$ & -0.01 & $(0.01)$ & & \\
\hline$\alpha_{214}$ & 0.00 & $(0.00)$ & & \\
\hline$\alpha_{215}$ & 0.01 & $(0.01)$ & & \\
\hline \multicolumn{5}{|c|}{ seasonal effect outflow } \\
\hline$\omega_{22}$ & $\overline{0.07}$ & $\overline{(0.01)}$ & 0.07 & $\overline{(0.01)}$ \\
\hline$\omega_{23}$ & 0.21 & $(0.01)$ & 0.20 & $(0.01)$ \\
\hline$\omega_{24}$ & 0.04 & $(0.01)$ & 0.04 & $(0.01)$ \\
\hline
\end{tabular}


Table 2: (Continued)

\begin{tabular}{|c|c|c|c|c|}
\hline \multicolumn{5}{|c|}{ cycle composition inflow } \\
\hline \multirow[t]{2}{*}{$\beta_{3}$} & \multicolumn{4}{|c|}{ (help wanted index) } \\
\hline & 0.69 & $(0.07)$ & 0.51 & $(0.06)$ \\
\hline \multicolumn{5}{|c|}{ seasonal effect composition inflow } \\
\hline$\omega_{32}$ & -0.05 & $(0.01)$ & -0.04 & $(0.01)$ \\
\hline$\omega_{33}$ & -0.11 & $(0.01)$ & -0.11 & $(0.01)$ \\
\hline$\omega_{34}$ & -0.09 & $(0.01)$ & -0.10 & $(0.01)$ \\
\hline \multicolumn{5}{|c|}{ interaction; duration parameters } \\
\hline$\overline{\xi_{10}}$ & -0.49 & $(0.41)$ & -0.91 & $(0.07)$ \\
\hline$\xi_{11}$ & 0.64 & $(0.52)$ & 1.10 & $(0.07)$ \\
\hline$\left(\xi_{11}\right)$ & -0.49 & $(0.41)$ & -0.91 & $(0.07)$ \\
\hline$\left(\xi_{12}\right)$ & -0.35 & $(0.30)$ & -0.72 & $(0.10)$ \\
\hline$\left(\xi_{13}\right)$ & 0.44 & $(0.38)$ & 0.56 & $(0.17)$ \\
\hline$\left(\xi_{14}\right)$ & 1.86 & $(1.53)$ & 2.95 & $(0.32)$ \\
\hline \multicolumn{5}{|c|}{ interaction; cycle parameters } \\
\hline$\overline{\alpha_{31}}$ & -0.12 & $(0.11)$ & & \\
\hline$\alpha_{32}$ & 0.01 & $(0.03)$ & & \\
\hline$\alpha_{33}$ & 0.05 & $(0.05)$ & & \\
\hline$\alpha_{34}$ & -0.03 & $(0.04)$ & & \\
\hline$\alpha_{35}$ & 0.01 & $(0.03)$ & & \\
\hline$\alpha_{36}$ & -0.02 & $(0.03)$ & & \\
\hline$\alpha_{37}$ & 0.05 & $(0.05)$ & & \\
\hline$\alpha_{38}$ & 0.06 & $(0.05)$ & & \\
\hline$\alpha_{39}$ & 0.05 & $(0.05)$ & & \\
\hline$\alpha_{310}$ & 0.02 & $(0.03)$ & & \\
\hline$\alpha_{311}$ & 0.06 & $(0.06)$ & & \\
\hline$\alpha_{312}$ & 0.05 & $(0.05)$ & & \\
\hline$\alpha_{313}$ & -0.00 & $(0.02)$ & & \\
\hline$\alpha_{314}$ & -0.01 & $(0.02)$ & & \\
\hline$\alpha_{315}$ & 0.07 & $(0.06)$ & & \\
\hline \multicolumn{5}{|c|}{ measurement errors } \\
\hline$\sigma_{b m}$ & 0.23 & $(0.00)$ & 0.24 & $(0.00)$ \\
\hline$\sigma_{b f}$ & 0.23 & $(0.00)$ & 0.24 & $(0.00)$ \\
\hline$\sigma_{w m}$ & 0.14 & $(0.00)$ & 0.15 & $(0.00)$ \\
\hline$\sigma_{w f}$ & 0.13 & $(0.00)$ & 0.14 & $(0.00)$ \\
\hline $\bar{N}$ & \multicolumn{2}{|c|}{$288 \times 4 \times 5$} & \multicolumn{2}{|c|}{$288 \times 4 \times 5$} \\
\hline $\log \mathcal{L}$ & \multicolumn{2}{|c|}{8180.53} & \multicolumn{2}{|c|}{7834.93} \\
\hline
\end{tabular}

Note: standard errors in parentheses. 
Figure 1: Help wanted index

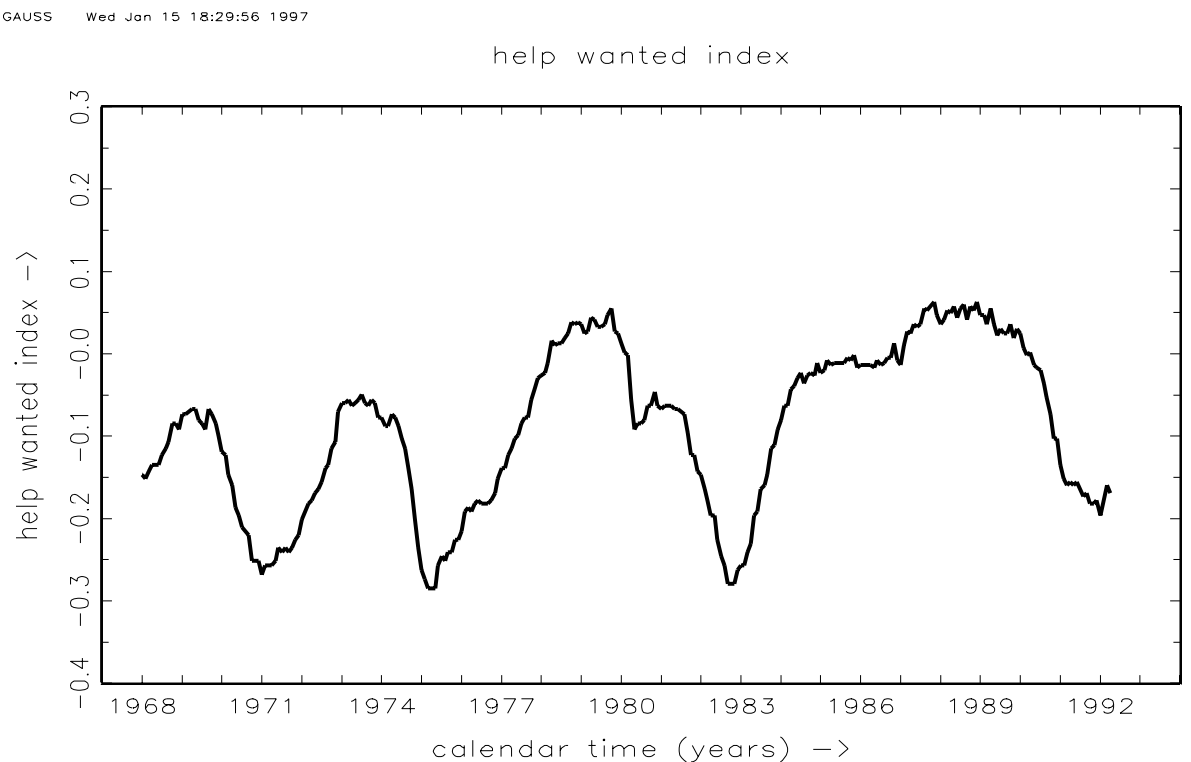

Figure 2: Estimated cycles in outflow and interaction

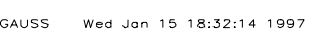

cycle

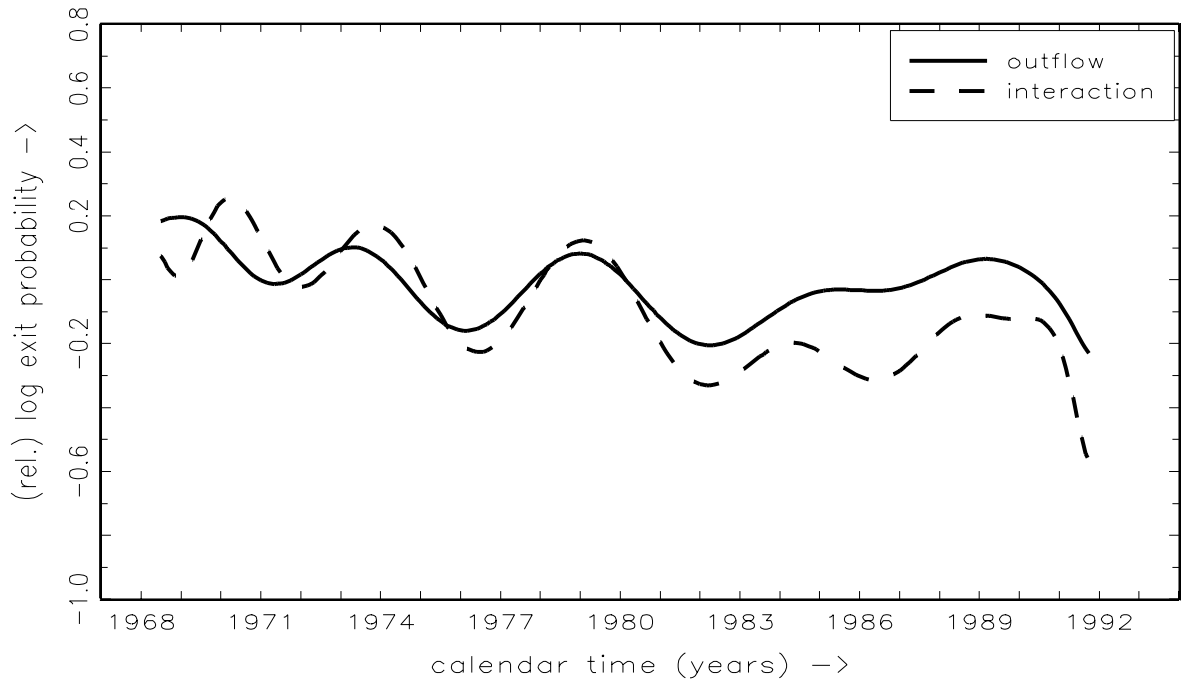


Figure 3: Cycles by duration

cycle

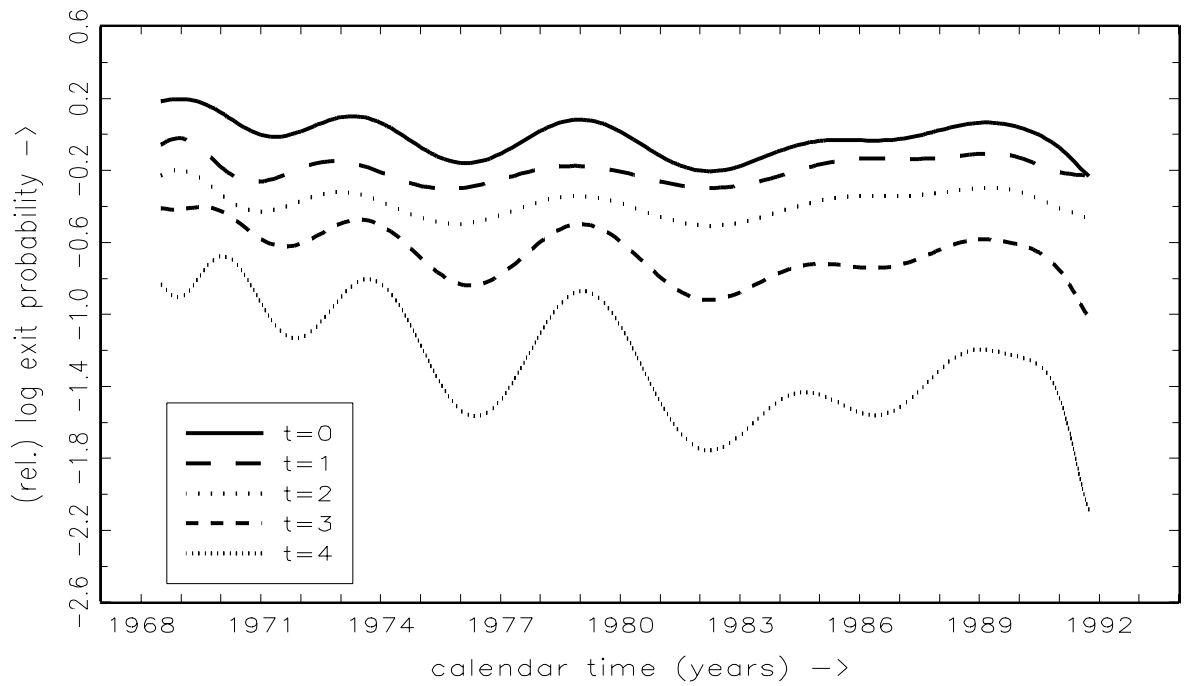

Figure 4: Duration dependence and the cycle

duration dependence

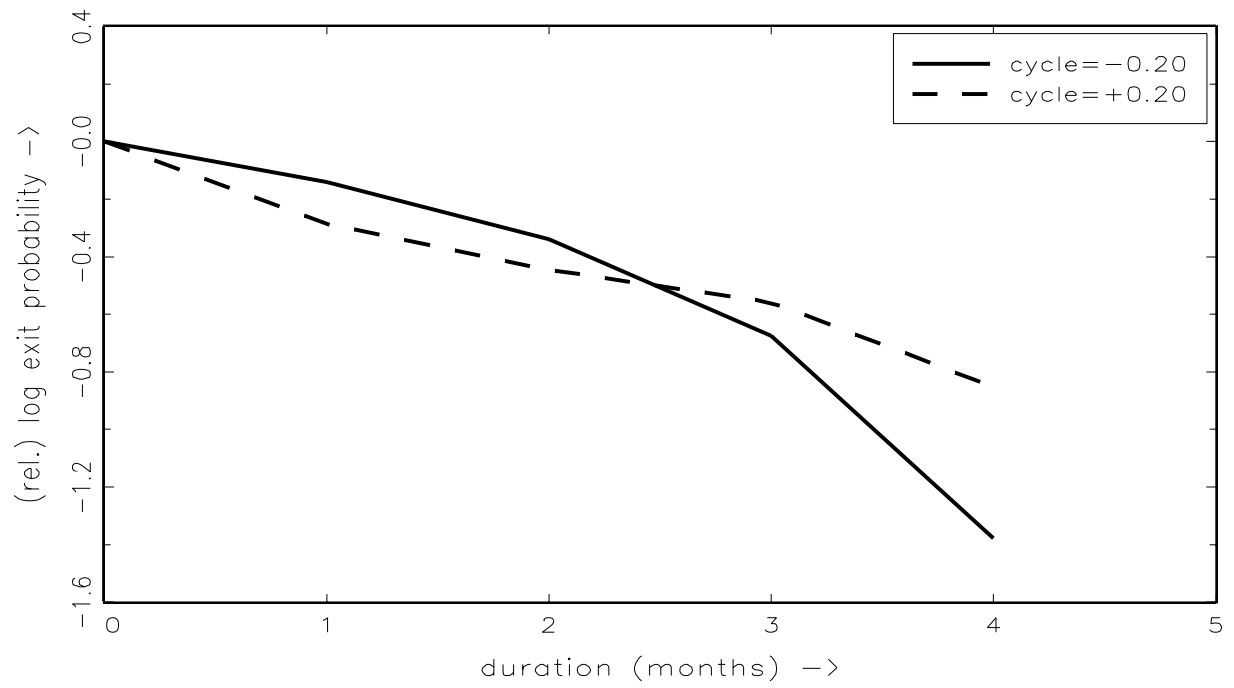


Figure 5: Cycle unemployment incidence rate by demographic group

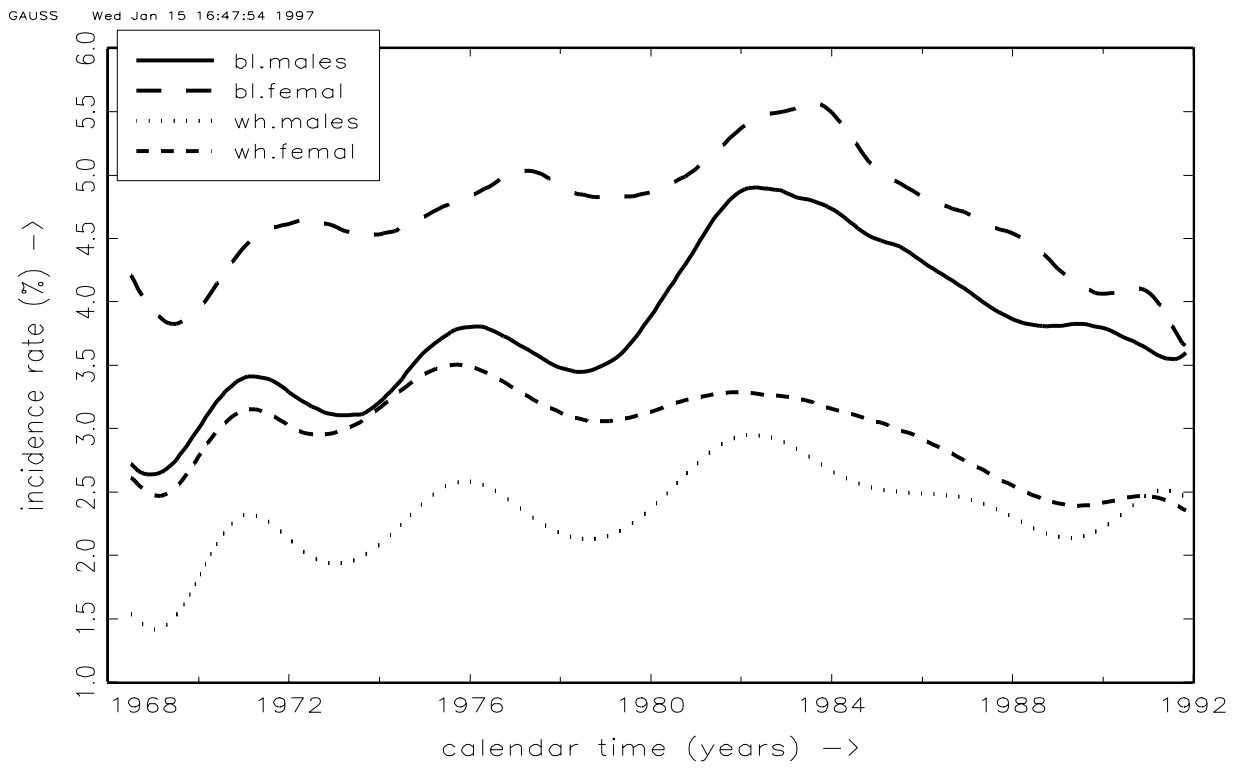

Figure 6: Unemployment rate; fitted series and data

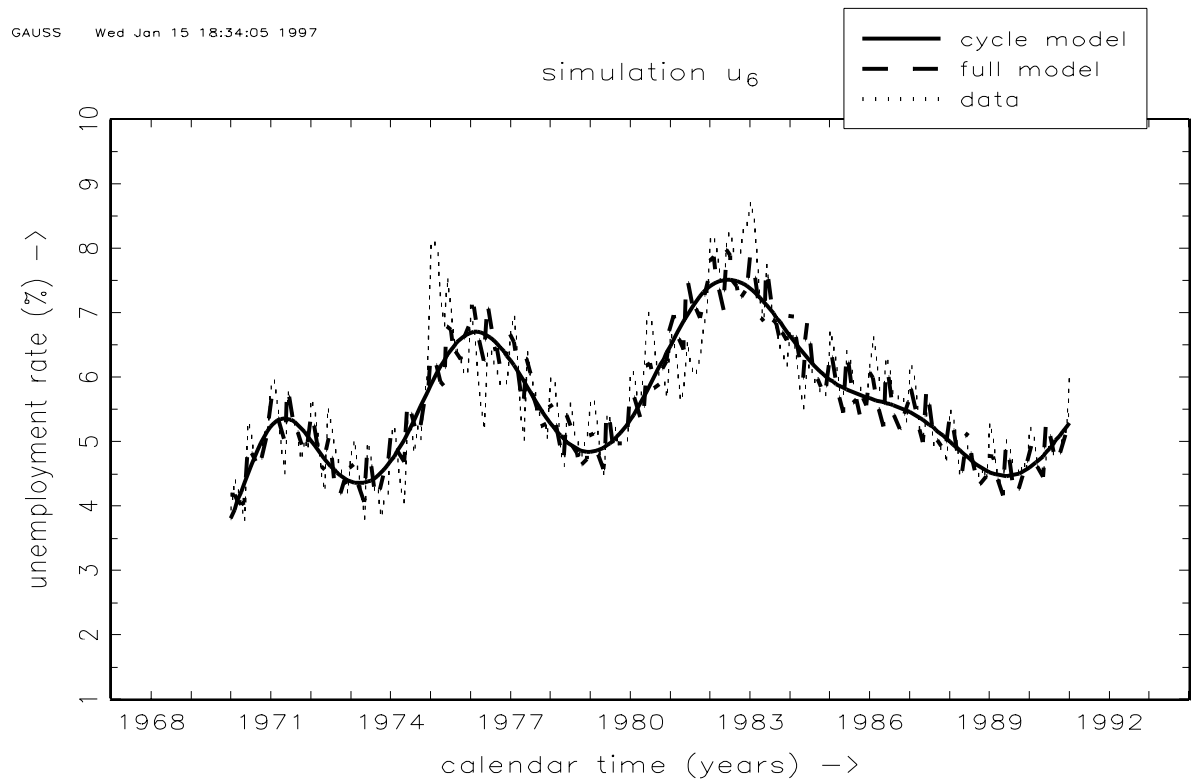


Figure 7: Unemployment rate; full cycle and without cycle outflow

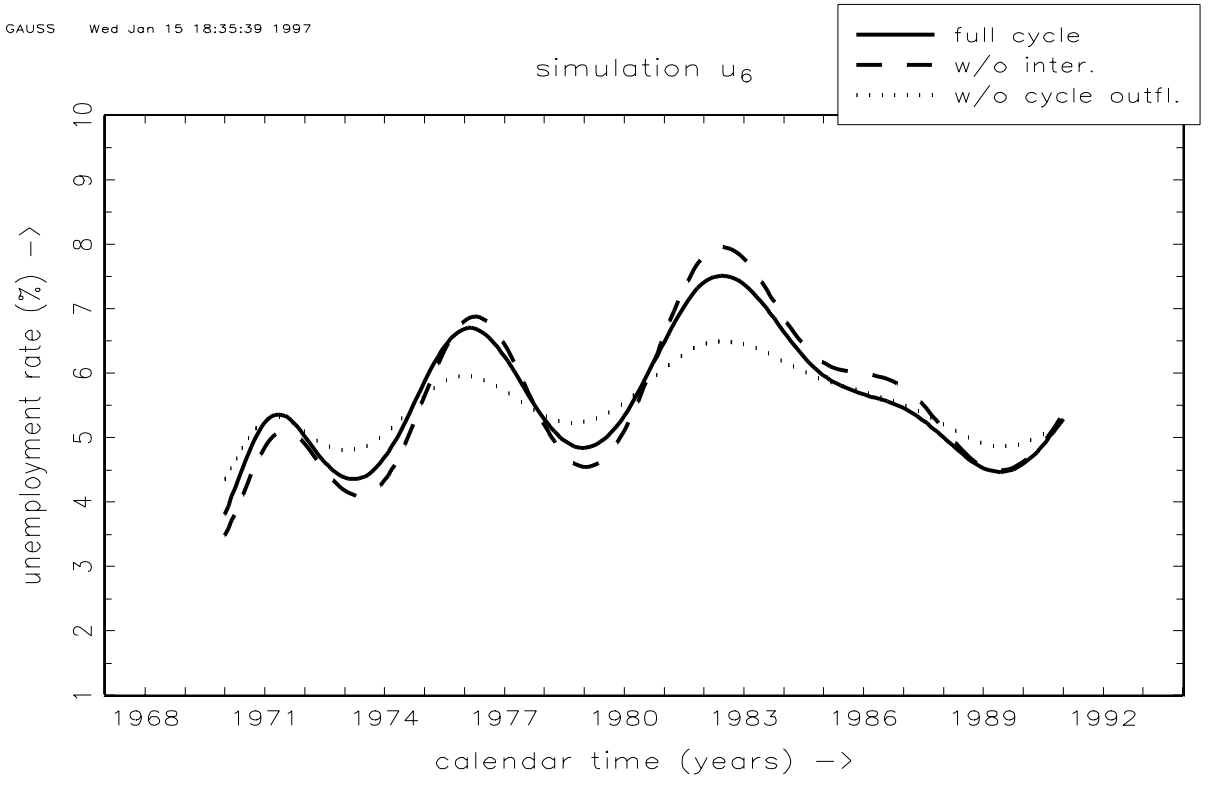

Figure 8: Unemployment rate; full cycle and without cycle incidence

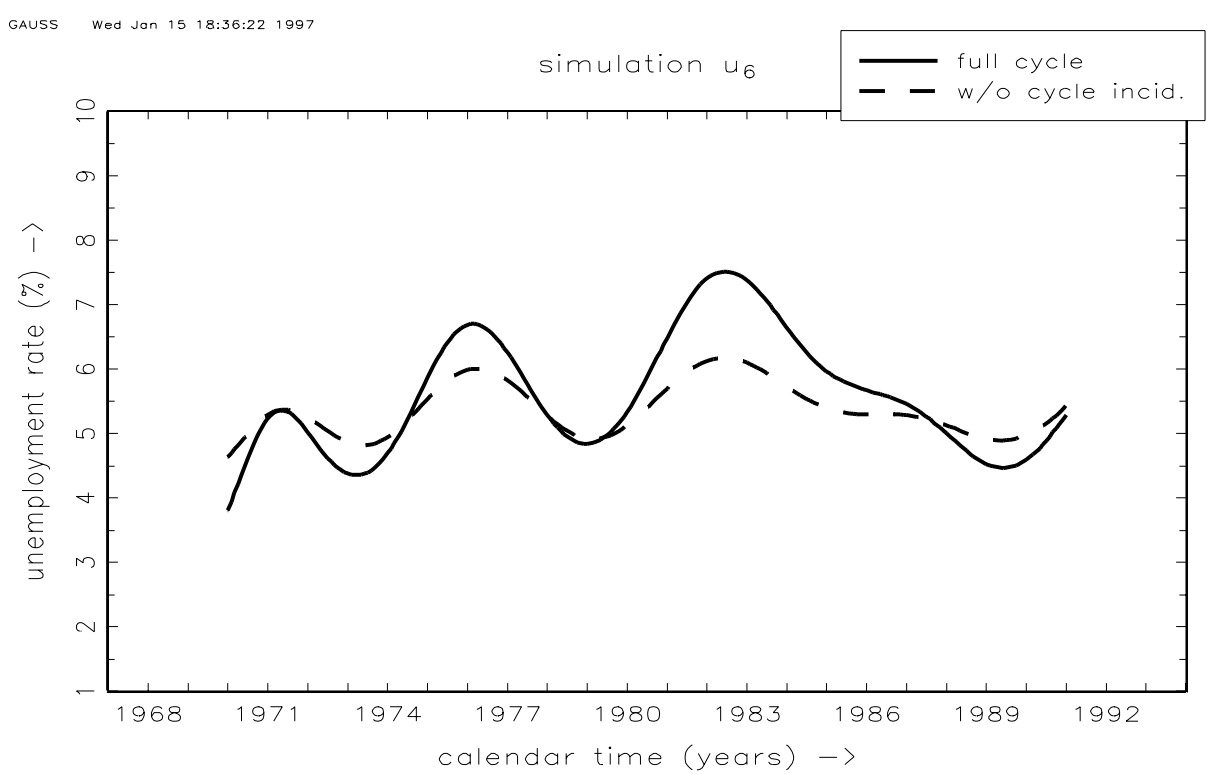


Figure 9: Unemployment rate; full cycle and without duration dependence

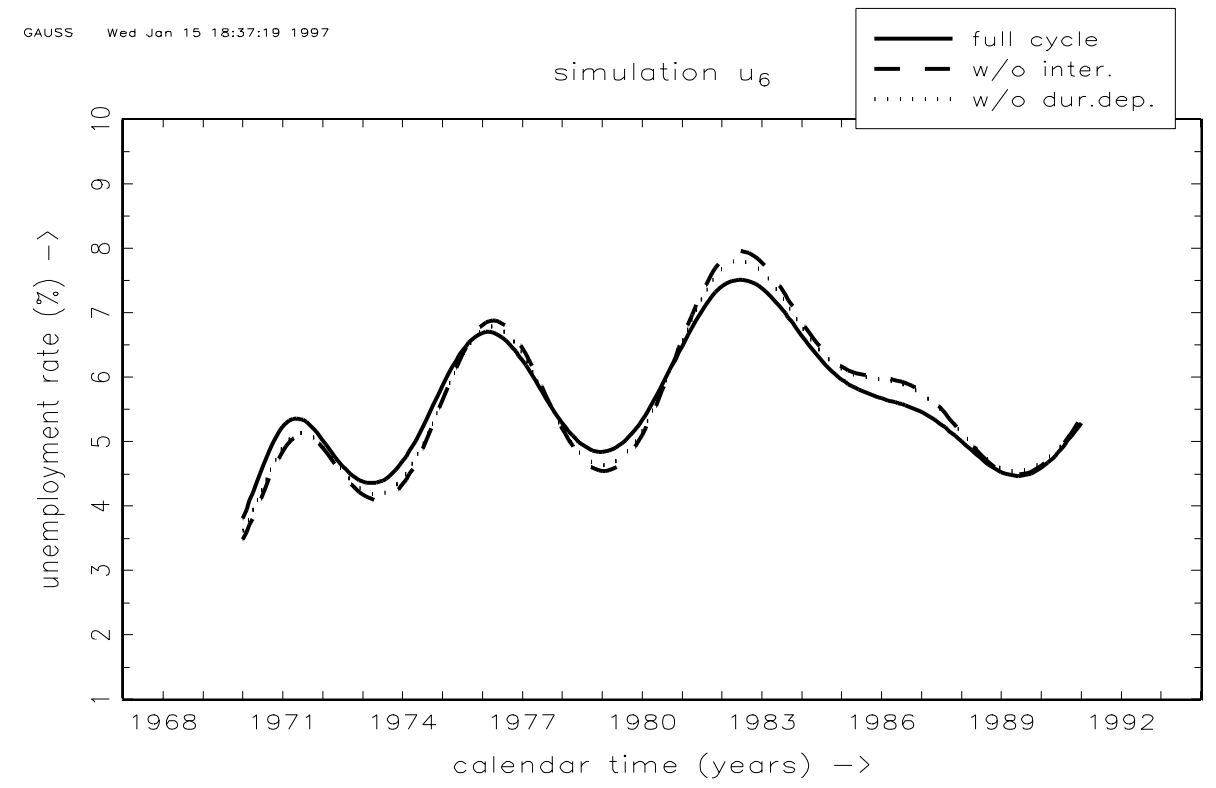

Figure 10: Unemployment rate; full cycle and with constant composition inflow

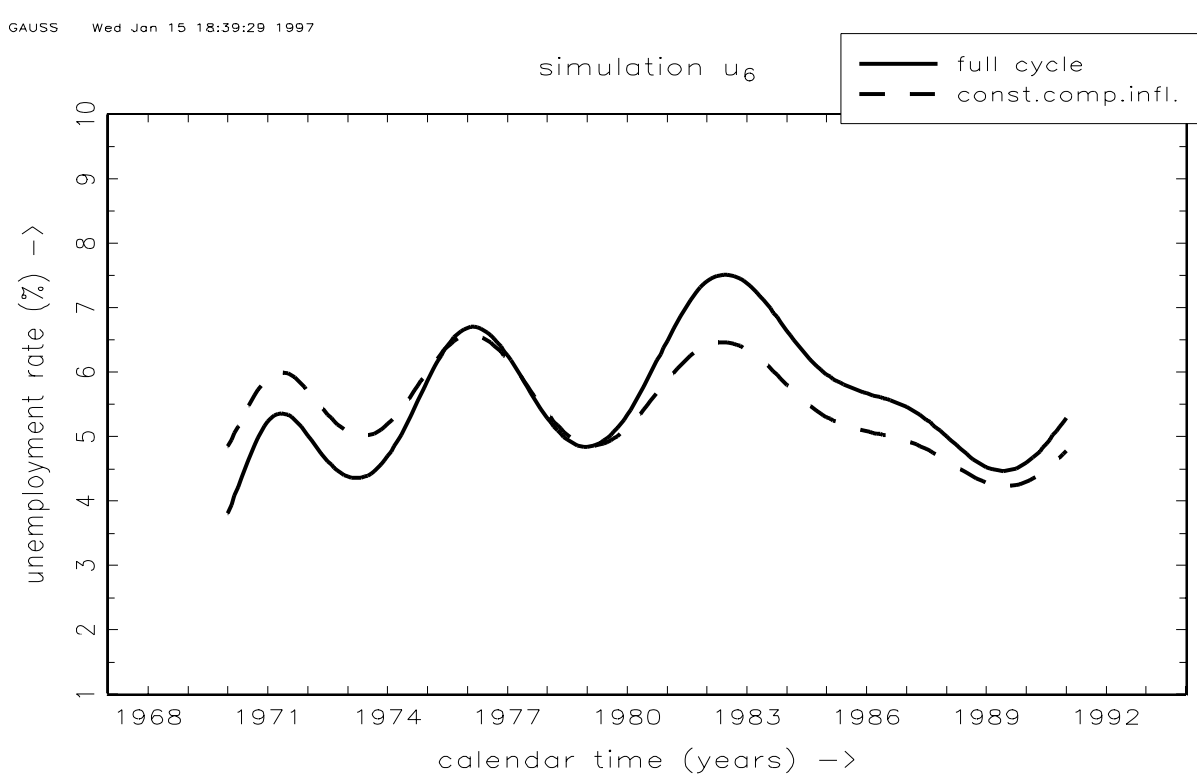

